Discussion Paper No. 895

\title{
NATURE OF COMPETITION AND NEW TECHNOLOGY ADOPTION
}

\author{
Krishnendu Ghosh Dastidar
}

March 2014

The Institute of Social and Economic Research Osaka University

6-1 Mihogaoka, Ibaraki, Osaka 567-0047, Japan 


\title{
Nature of competition and new technology adoption*
}

\author{
Krishnendu Ghosh Dastidar \\ Jawaharlal Nehru University, New Delhi, India. \\ Email: krishnendu.dastidar@gmail.com, kgd12@yahoo.com
}

March, 2014

\begin{abstract}
This paper analyses the incentives to adopt cost-reducing technology by firms in a horizontally differentiated industry. In our model there are several suppliers of a new technology. The extent of the cost reduction depends on the quality of the new technology. A firm has to buy the technology in a 'scoring auction'. This means that both the price and the quality (which affects marginal cost of production) of this new technology are no longer given but depend on the equilibrium outcome in the 'scoring auction'. We show that the nature of competition (Cournot or Bertrand) has no effect on the equilibrium decision of the firms to adopt the new technology when the quality of the new technology offered by the suppliers lies in the interior of the feasible range of qualities. In this case, both firms adopt new technology. However, when there is a corner solution, then it is possible to have equilibria where only one firm (or no firm) adopts the new technology. With corner solution the nature of competition (Cournot or Bertrand) makes a difference to the equilibrium outcomes.
\end{abstract}

Journal of Economic Literature No. L13, L11, D43, D44

\section{Introduction}

Consider a differentiated product duopoly. The firms have the option of investing in a new technology which is cost-reducing. This may be a new machine which brings down marginal cost of production (increases efficiency) or a new software which improves product design and effectively reduces costs. The extent of the reduction in marginal cost depends on the quality of the new technology. The following questions arise:

1. Will the firms adopt this cost-reducing new technology?

2. Does the nature of competition (Cournot or Bertrand) matter?

In the industrial organisation literature the above questions have been addressed in various ways. However, our approach will be different. In order to find answers to the above questions we consider the following two-stage game.

*I am indebted to Masaki Aoyagi, Vijay Kaul, Noriaki Matshushima, Toshihiro Matsumura, Achintya Ray and Makoto Yano for very helpful comments. Comments from the participants at the Aomori Kyoto International Workshop and seminar participants at Tokyo University were also very helpful. The paper was written when I was a 'Visiting Research Scholar' at the Institute of Social and Economic Research, Osaka University in 2013-14. ISER provided me with excellent research facilites and stimulating intellectual ambience and I am very grateful for that. The usual disclaimer applies. 
1. In the first stage firms simultaneously and independently decide whether or not to adopt the new technology. There are several suppliers of this new technology. The quality of the new technology lies in the interval $[0, \bar{x}]$. Higher is the quality of the new technology, the lower will be the marginal cost of production. The extent of reduction in marginal cost depends on the effectiveness of the new technology in reducing marginal cost. If a firm decides to adopt the new technology, it has to purchase it in a scoring auction. This means that both the cost of buying this new technology and the quality of the technology (which affects the marginal cost of production) are no longer given but they depend on the equilibrium outcome in the scoring auction.

2. In the second stage the firms engage either in Cournot competition or in Bertrand competition in a differentiated product market. The mode of product market competition, Cournot or Bertrand, in the second stage is exogenously determined. The outcome of the scoring auction is revealed to both the firms before they engage in the second stage duopoly competition. This means that when a firm engages in second stage competition (Cournot or Bertrand) it knows the quality choice (and hence the marginal cost) of its rival.

We find that the equilibrium outcome crucially depends on whether or not the quality of the new technology offered by the suppliers lies in the interior of $[0, \bar{x}]$.

In sharp contrast to the related results in the literature we show that both firms choose to adopt the new technology in the first stage when the quality of the new technology offered by the suppliers lies in the interior of $[0, \bar{x}]$. This is true regardless of the value of differentiation parameter and regardless of the nature of second stage competition (Cournot or Bertrand). However, when there is a corner solution (where all the suppliers offer quality $\bar{x}$ ) then it is possible to have an equilibrium where only one firm (or no firm) adopts the new technology. With corner solution in quality, in some cases the nature of competition (Cournot or Bertrand) makes a difference to the equilibrium outcomes. We show that this depends on the degree of product differentiation and the expected cost of acquiring the new technology. It may be noted that with corner solution, our results are somewhat closer to the existing ones in the literature.

The novelty of our paper is that we use 'scoring auction' as a mechanism to buy new technology. In all papers in the related literature firms adopt the new technology either by incurring a fixed cost (as in Bester and Petrakis (1993), Pal (2010) and Elberfeld and Nti (2004)) or by participating in a 'standard auction' (as in Das-Verma (2003) and Moldovanu and Sela (2003)).

Why Scoring auction? In the traditional theory of standard procurement auctions, the buyer (auctioneer) cares only about the price of the object, but not the other attributes. ${ }^{1}$ However, in many procurement situations, the buyer cares about attributes other than price when evaluating the offers submitted by suppliers. Non-monetary attributes that buyers care about include quality, time to completion etc. For example, in the contract for the construction of a new aircraft, the specification of its characteristics is probably as important as its price (see Branco, 1997). Under these circumstances, auctions are usually multidimensional: bidders submit bids with the relevant characteristics of the project (among which is price), then the procurement agency gives a score to each bid and makes its decisions based on these scores. The essential element of such multidimensional auctions is a scoring rule. For example, the Department of Defence in USA often relies on competitive source selection to procure weapon systems (see Che, 1993). Each individual

\footnotetext{
${ }^{1}$ It may be noted that the standard benchmark model of auctions is really a price-only auctions. See Krishna (2010) for all the major results in the standard model.
} 
component of a bid of the weapon system is evaluated and assigned a score, these scores are summed to yield a total score, and the firm achieving the highest score wins the contract.

In our set-up any firm which is planning to buy the new technology cares both about the price of the new technology and the quality that is offered. The reason is simple: both the price and quality of the new technology affects the profit of the firm. The price of the new technology is like a fixed cost and the quality of the new technology directly affects the marginal cost of production. For example, if the firms are airline companies then they may invest in new aircraft with fuel efficient engines. The more fuel efficient the engines of the new aircraft are, the more will be the savings in fuel costs (which effectively means that marginal cost of operating a flight comes down). Such airline companies evaluate offers from suppliers of new aircraft based on not only the prices of the aircraft but also on the quality of new fuel efficient engines. In a country like India where fuel costs are very high, airlines greatly value the fuel cost savings. Airline companies in India typically purchase new aircraft after evaluating competing offers (that include price as well as various quality parameters) from big aircraft suppliers like Boeing and Airbus. ${ }^{2}$

We now proceed to provide a brief discussion of the related papers in the literature and show how our exercise differs from these papers.

The related literature Our two stage game is somewhat close to the approach in Bester and Petrakis (1993) and Pal (2010). In these two papers the basic model is as follows. First, one firm (or both firms) decides (decide) whether or not to adopt a cost-reducing technology, by incurring some given cost. If a firm adopts a cost-reducing technology, the reduction in marginal cost is discrete and known. Next, the firms engage either in Cournot competition or in Bertrand competition. ${ }^{3}$ Bester and Petrakis (1993) show that Cournot competition provides a stronger incentive to innovate than Bertrand competition if the degree of product differentiation is low, and a weaker incentive if this degree is high. Pal (2010) shows that, if the given fixed cost of the technology is high, Bertrand competition provides a stronger incentive to adopt technology than Cournot competition unless the given cost-reducing effect of the technology is very low. On the contrary, if the cost of the technology is low, Cournot competition fares better than Bertrand competition in terms of technology adoption by firms.

Note that in the above papers firms have to incur some given fixed cost to adopt the new technology and the reduction in marginal cost is also given. That is, both the price of the new technology and the associated reduction in marginal cost are known to the firms in the beginning. In contrast, in our model, neither the price of the new technology nor the reduction in marginal cost are known to the firms at the start. Firms come to know of these only after the outcome of the scoring auction has been revealed.

Another set of papers (for example, Elberfeld and Nti, 2004) deal with the case where the reduction in marginal cost is itself uncertain. Here a firm may either undertake an up-front in-

\footnotetext{
${ }^{2}$ For example in 2011, after evaluating competing offers, IndiGo (a low-cost Indian airline) ordered 180 Airbus A320s from Airbus for a valuation of $\$ 15.6$ billion. Earlier India's national carrier, Air India, had placed an order for 111 Boeing and Airbus aircraft for $\$ 11$ billion in 2005. As Airbus offers more fuel-efficient aircraft, in Indian aviation market the demand for its aircarfts is increasing. Boeing's market share has slumped in the Indian market and Airbus now controls about $73 \%$ of the Indian pie. See Keller (2011) and Singhal (2011) for the information regarding the order for Airbus aircrafts placed by some of the Indian airline companies.

${ }^{3}$ Boone (2001) analyses the effects of intensity of product market competition on R\&D incentives in a model capturing the strategic interactions between firms with different cost levels. Bonano and Haworth (1998) analyses the relationship between intensity of competition and the profitability of innovative activity. In a context where there is conflict between static and dynamic efficiency, Delbono and Denicolo (1990) compare the equilibrium R\&D investment under Bertrand and Cournot competition in a symmetric and homogeneous oligopoly.
} 
vestment to adopt the new technology with low marginal cost, or it may continue to use the old technology with high marginal cost. The cost of adoption of such a new technology is given. After technological commitments have been made, uncertainty in marginal cost is resolved, and the firms play a Cournot oligopoly game. ${ }^{4}$ It is shown that if in equilibrium both technologies are employed, more uncertainty about the new technology increases (decreases) the number of innovating firms and decreases (increases) the product price if the up-front investment is large (small). In Elberfeld and Nti (2004) the second stage competition is Cournot only and consequently, the issues regarding differences due to nature of competition (Cournot or Bertrand) do not arise.

Note that in Elberfeld and Nti (2004) the cost of the new technology is given but the reduction in marginal cost (due to adoption of new technology) is uncertain. In contrast, in our model, before the outcome of the scoring auction is known, both the cost of the new technology and the quality of the new technology (and consequently the reduction in marginal cost) are uncertain.

There is yet another set of papers in the literature in which there is first an auction for a patent and then the equilibrium outcome of this auction influences the future interaction (oligopoly game) among agents. For example, in Das-Verma (2003) and Moldovanu and Sela (2003) a costreducing process innovation protected by a patent is sold to one of several firms. The firms bid non-cooperatively for this process innovation. Following the auction, in the second stage of the game, the firms compete against each other in an imperfectly competitive product market. The process innovation won from the first stage auction lowers the winning bidder's cost in the product market. The value of the patent for a firm is the difference between the profit it makes in case it acquires the patent, and the profit in case it does not. ${ }^{5}$ Das-Verma (2003) examines whether a first-price, sealed-bid auction is efficient in allocating a process innovation amongst oligopolists engaged in either Cournot or Bertrand competition and shows that for Cournot, there exists a symmetric, unique equilibrium in strictly increasing strategies (that are allocatively efficient). For Bertrand, such an efficient equilibrium may not exist. The paper by Moldovanu and Sela (2003) considers several firms engaged in price competition under conditions of asymmetric information about production costs. Incomplete information about production costs yields an auction model with both private and common value components. The main result in this paper is that standard auction mechanisms lead to inefficient allocations.

Like Das-Verma (2003) and Moldovanu and Sela (2003) we have an auction prior to product market competition. But ours is a scoring auction whereas these papers use a standard auction. In these papers there is a reduction only in the winning bidder's cost in the product market. In our set-up any firm can choose to reduce its marginal cost by purchasing the new technology in a scoring auction. The new technology is not protected by any patent. Hence, in our model it is possible to have a scenario where both firms reduce marginal costs by adopting the new technology. Moreover, unlike Das-Verma (2003) and Moldovanu and Sela (2003), in our model the reduction in marginal cost does not depend on firms' types but on the quality of the new technology.

Plan of the paper In section 2 we provide the model of our exercise. Section 3 gives the equilibrium analysis of scoring auction. In section 4 we discuss the second-stage equilibrium in the product market. Section 5 provides our major results. We analyse whether in equilibrium, the firms choose the new technology or not. We do so both for the case where the quality of the new technology offered by the suppliers lies in the interior of $[0, \bar{x}]$ and for the case when there is a

\footnotetext{
${ }^{4}$ In a different approach, Jensen (1992) examines the welfare effects of adopting an innovation when there is uncertainty about whether it will succeed or fail.

${ }^{5}$ Note that we will not be dealing with patent races. There is a huge literature on patent races (starting with the classic papers by Dasgupta and Stiglitz, 1980 and Loury, 1979).
} 
corner solution (where the suppliers offer quality $\bar{x}$ ). Section 6 provides some concluding remarks. Lastly, the appendix provides the proofs of most of our propositions.

We now proceed to provide the model of our exercise.

\section{The Model}

We consider the following two-stage game.

Stage 1 : Firm 1 and firm 2 simultaneously and independently decide whether or not to adopt the new technology. There are $n$ suppliers of the new technology. A firm has to buy the technology in a 'scoring auction'.

Stage 2 : Firms 1 and 2 engage either in Cournot competition or in Bertrand competition in a differentiated product market.

First stage scoring auction A firm $i$ which decides to adopt the new technology solicits bids from $n$ suppliers. Each bid is two-dimensional. It specifies an offer of promised quality, $x$ and price, $\phi$, at which a fixed quantity of products with the offered level of quality $x$ is delivered. The quantity is normalised to one. For simplicity quality is modelled as a one-dimensional attribute. The quality of the technology is $x$ and $x \in[0, \bar{x}]$.

The cost of supplying the new technology is given by $\psi\left(x, \theta_{i}\right)=x \theta_{i}$ where $\theta_{i}$ is the type of the supplier $i$.

Prior to bidding each supplier $i$ learns its cost parameter $\theta_{i}$ as private information. The buyer and other suppliers (i.e. other than supplier $i$ ) do not observe $\theta_{i}$ but only knows the distribution function of the cost parameter. It is assumed that $\theta_{i}$ is identically and independently distributed over $[\underline{\theta}, \bar{\theta}]$ where $0<\underline{\theta}<\bar{\theta}$. The distribution function of $\theta_{i}$ is given by $F($.$) and the density$ function by $f($.$) .$

Let $S(x, \phi)=s(x)-\phi$ denote a scoring rule for an offer $(x, \phi)$, where $s($.$) is strictly increasing$ in $x$. The rule is assumed to be publicly known to the suppliers of the new technology at the start of bidding. In our model $s(x)$ is equal to the second-stage gross profit of the firm seeking the new technology. This profit depends on (i) the nature of the second stage competition (Cournot or Bertrand) and on (ii) whether the firm and its rival have chosen the new technology or not. Also, in our model $\phi$ is the amount paid by the technology seeking firm to the supplier winning the contract. That is, $\phi$ is like a fixed cost of purchasing the technology. Consequently, the second-stage net profit of the firm is $s(x)-\phi$.

The firm awards the contract to a supplier whose offer achieves the highest score. This is similar to a standard auction. In this paper we will model only first-score auction. In a first-score auction the winning supplier's offer is finalised as the contract. ${ }^{6}$

Second-stage duopoly competition For the second-stage competition we consider a representative consumer's utility function based on Dixit (1979). Scores of papers in the literature have used this. A small sample of such papers is as follows: Singh and Vives (1984), Hackner ( 2000), Bester and Petrakis (1993), Zanchettin (2006) and Pal (2010).

\footnotetext{
${ }^{6}$ This auction rule is a two-dimensional analogue of the first-price auction. In a second-score auction the winning supplier is required to match the highest rejected score. In meeting this score, the supplier is free to choose any quality-price combination. See Che (1993) for the details.
} 
On the demand side of the market, the representative consumer's utility function of two differentiated products, $q_{1}$ and $q_{2}$, and a numeraire good, $q_{0}$ is given by

$$
U=a\left(q_{1}+q_{2}\right)-\frac{1}{2}\left(q_{1}^{2}+q_{2}^{2}+2 \gamma q_{1} q_{2}\right)+q_{0} .
$$

The parameter $\gamma$ measures the degree of product differentiation. We consider the case of substitute goods: $0<\gamma<1$. Lower value of $\gamma$ denotes higher degree of product differentiation, i.e., lower degree of substitutability between products. Note that when $\gamma$ is close to unity then the products are nearly homogeneous (perfect substitutes) and when $\gamma$ is close to zero the products are nearly independent

The utility function generates the system of linear demand functions

$$
q_{i}=\frac{1}{1-\gamma^{2}}\left[a(1-\gamma)-p_{i}+\gamma p_{j}\right] ; i, j=1,2 ; i \neq j
$$

The inverse demand functions are

$$
p_{i}=a-q_{i}-\gamma q_{j} ; i, j=1,2 ; i \neq j
$$

Initially (before the adoption of new technology) the marginal cost of production of firms 1 and 2 is $c$.

We assume the following: (i) $a>2 c$ (ii) $\gamma \in(0, \underline{\gamma})$ where $\underline{\gamma}=\frac{-a+\sqrt{9 a^{2}+8 c^{2}-16 a c}}{2(a-c)}$. This ensures that both firms produce strictly positive output in equilibrium in Cournot competition as well as in Bertrand competition for all possible cases.

If firm 1 adopts the new technology of quality $x$ its marginal cost reduces to $c(1-k \lambda(x))$ where $k \geq 1, \lambda(0)=0, \lambda^{\prime}(x)>0$ for all $x>0$ and $k \lambda(x) \leq 1$ for all $x$. If firm 2 adopts the new technology of quality $x$ its marginal cost reduces to $c(1-\lambda(x))$. Note that $\lambda(x)$ denotes the effectiveness of the new technology in reducing marginal cost. Since $k \geq 1$ firm 1 is more competent at reducing marginal cost. Note that $x=0$ is equivalent to not adopting the new technology.

Before giving our main results we need to provide some preliminaries on order statistics.

\subsection{Order Statistics : some notations and preliminaries}

Let $y_{1}, y_{2} . . y_{n}$ denote a random sample of size $n$ drawn from $F($.$) . Then t_{1} \leq t_{2} \ldots \leq t_{n}$ where $t_{i} s$ are $y_{i} s$ arranged in increasing magnitudes, are defined to be the order statistics corresponding to the random sample $y_{1}, y_{2} \ldots y_{n}$.

We would be interested in $t_{1}$ (lowest order statistic) and $t_{2}$ (second lowest order statistic). The corresponding distribution functions and density functions are $F_{1}(),. F_{2}($.$) and f_{1}(),. f_{2}($.$) .$ 
Note that

$$
\begin{aligned}
F_{1}(t) & =1-(1-F(t))^{n} \\
\text { and } F_{2}(t) & =1-(1-F(t))^{n}-n F(t)(1-F(t))^{n-1} \\
f_{1}(t) & =n(1-F(t))^{n-1} f(t) \\
\text { and } f_{2}(t) & =n(n-1) F(t)(1-F(t))^{n-2} f(t)
\end{aligned}
$$

\section{Equilibrium in scoring auction}

Each auction rule can be viewed as a Bayesian game where each supplier picks a quality-price combination $(x, \phi)$ as a function of its cost parameter. We now provide the first result (from Che, 1993).

Lemma 1 A unique symmetric equilibrium of a first-score auction is one in which each supplier with type $\theta$ offers

$$
\begin{aligned}
& x_{s}(\theta)=\arg \max _{x \in[0, \bar{x}]} s(x)-x \theta \\
& \phi_{s}(\theta)=x_{s}(\theta) \theta+\int_{\theta}^{\bar{\theta}} x_{s}(t)\left[\frac{1-F(t)}{1-F(\theta)}\right]^{n-1} d t
\end{aligned}
$$

remark 1 Note that since $S\left(x_{s}(\theta), \phi_{s}(\theta)\right)=s\left(x_{s}(\theta)\right)-\phi_{s}(\theta)$ is strictly decreasing in $\theta$ the firm with lowest type $(\theta)$ wins the contract in equilibrium ${ }^{7}$.

We will assume that $x_{s}(\theta)>0$ for all $\theta \in[\underline{\theta}, \bar{\theta}]$. This ensures that even the least efficient supplier (whose type is $\bar{\theta}$ ) of the new technology quotes positive quality index in equilibrium.

The expected payoff to firm $i$ which chooses the new technology is the expected value of $(s(x)-\phi)$, where $s(x)$ is the second-stage profit. Now using lemma 1 we get

$$
\begin{aligned}
& \operatorname{Exp} .(s(x)-\phi)=\int_{\underline{\theta}}^{\bar{\theta}}\left(s\left(x_{s}(\theta)\right)-\phi_{s}(\theta)\right) f_{1}(\theta) d \theta \\
= & \int_{\underline{\theta}}^{\bar{\theta}}\left(s\left(x_{s}(\theta)\right)-x_{s}(\theta) \theta-\int_{\theta}^{\bar{\theta}} x_{s}(t)\left[\frac{1-F(t)}{1-F(\theta)}\right]^{n-1} d t\right) f_{1}(\theta) d \theta
\end{aligned}
$$

We now provide our next result which also follows from Che (1993). In the appendix we provide a much simpler proof.

Lemma 2 The expected payoff to firm $i$ in a first-score auction is as follows:

$$
\begin{aligned}
& \int_{\underline{\theta}}^{\bar{\theta}}\left(s\left(x_{s}(\theta)\right)-x_{s}(\theta) \theta-\int_{\theta}^{\bar{\theta}} x_{s}(t)\left[\frac{1-F(t)}{1-F(\theta)}\right]^{n-1} d t\right) f_{1}(\theta) d \theta \\
= & \int_{\underline{\theta}}^{\bar{\theta}}\left[s\left(x_{s}(\theta)\right)-x_{s}(\theta) \theta\right] f_{2}(\theta) d \theta
\end{aligned}
$$

We will need lemma 2 to prove some of our main results.

\footnotetext{
${ }^{7}$ See Che (1993) for the formal demonstration.
} 


\section{Equilibrium in the second stage}

The mode of product market competition, Cournot or Bertrand, in the second stage is exogenously determined. The outcome of the scoring auction (held in the first stage) is revealed to both the firms before they engage in the second stage duopoly competition. This means that when a firm engages in second stage competition (Cournot or Bertrand) it knows the quality choice (and hence the marginal cost) of its rival.

At the end of first-stage there are three possible cases : (i) no firm has adopted the technology (ii) only one firm, either firm 1 or firm 2, has adopted the technology (iii) both of them have adopted the technology.

We use the following notations for equilibrium outcomes ('C' stands for 'choose the new technology' and 'D' stands for 'don't choose'):

1. $(C, C)$ : firm 1 and firm 2 both adopt the new technology

2. $(C, D)$ : firm 1 adopts the new technology but firm 2 does not

3. $(D, C)$ : firm 2 adopts the new technology but firm 1 does not

4. $(D, D)$ : both firms do not adopt the new technology

\subsection{Cournot competition}

We first analyse the case when there is quantity competition in the second stage. Let $x_{i}$ and $\phi_{i}$ respectively be the equilibrium quality obtained and the payment made by firm $i$ in the first-stage if it adopts the technology.

Cournot quantities and profits are denoted by $q_{i}$ and $\pi_{i}$ respectively.

Note that

$$
\begin{aligned}
& x_{1}^{D C}=x_{1}^{D D}=0 \text { and } \\
& \phi_{1}^{D C}=\phi_{1}^{D D}=0 .
\end{aligned}
$$

Similarly

$$
\begin{aligned}
& x_{2}^{C D}=x_{2}^{D D}=0 \text { and } \\
& \phi_{2}^{C D}=\phi_{2}^{D D}=0 .
\end{aligned}
$$

The equilibrium outcomes in stage 2 in the case of quantity competition in the product market, given the technology adoption decisions of firms, are as follows. 
1. When no firm adopts the new technology:

$$
\begin{aligned}
q_{i}^{D D} & =\frac{(a-c)(2-\gamma)}{\left(4-\gamma^{2}\right)} \\
\pi_{i}^{D D} & =\left[q_{i}^{D D}\right]^{2}
\end{aligned}
$$

2. When both firms adopt the new technology:

$$
\begin{aligned}
q_{1}^{C C} & =\frac{(a-c)(2-\gamma)+c\left(2 k \lambda\left(x_{1}^{C C}\right)-\gamma \lambda\left(x_{2}^{C C}\right)\right)}{\left(4-\gamma^{2}\right)} \\
\pi_{1}^{C C} & =\left[q_{1}^{C C}\right]^{2}-\phi_{1}^{C C} \\
q_{2}^{C C} & =\frac{(a-c)(2-\gamma)+c\left(2 \lambda\left(x_{2}^{C C}\right)-\gamma k \lambda\left(x_{1}^{C C}\right)\right)}{\left(4-\gamma^{2}\right)} \\
\pi_{2}^{C C} & =\left[q_{2}^{C C}\right]^{2}-\phi_{2}^{C C}
\end{aligned}
$$

3. When firm 1 adopts the new technology but firm 2 does not:

$$
\begin{aligned}
q_{1}^{C D} & =\frac{(a-c)(2-\gamma)+2 c k \lambda\left(x_{1}^{C D}\right)}{\left(4-\gamma^{2}\right)} \\
\pi_{1}^{C D} & =\left[q_{1}^{C D}\right]^{2}-\phi_{1}^{C D} \\
q_{2}^{C D} & =\frac{(a-c)(2-\gamma)-c k \lambda\left(x_{1}^{C D}\right) \gamma}{\left(4-\gamma^{2}\right)} \\
\pi_{2}^{C D} & =\left[q_{2}^{C D}\right]^{2}
\end{aligned}
$$

4. When firm 2 adopts the new technology but firm 1 does not:

$$
\begin{aligned}
q_{1}^{D C} & =\frac{(a-c)(2-\gamma)-c \lambda\left(x_{2}^{D C}\right) \gamma}{\left(4-\gamma^{2}\right)} \\
\pi_{1}^{D C} & =\left[q_{1}^{D C}\right]^{2} \\
q_{2}^{D C} & =\frac{(a-c)(2-\gamma)+2 c \lambda\left(x_{2}^{D C}\right)}{\left(4-\gamma^{2}\right)} \\
\pi_{2}^{D C} & =\left[q_{2}^{D C}\right]^{2}-\phi_{2}^{D C}
\end{aligned}
$$

\subsection{Bertrand competition}

We now analyse the case when there is price competition in the second stage. Let $y_{i}$ and $\Phi_{i}$ respectively be the equilibrium quality obtained and the payment made by firm $i$ in the first-stage if it adopts the technology. Bertrand prices, quantities and profits are denoted by $p_{i}, b_{i}$ and $B_{i}$ respectively. 
Note that

$$
\begin{aligned}
y_{1}^{D C} & =y_{1}^{D D}=0 \text { and } \\
\Phi_{1}^{D C} & =\Phi_{1}^{D D}=0 .
\end{aligned}
$$

Similarly

$$
\begin{aligned}
y_{2}^{C D} & =y_{2}^{D D}=0 \text { and } \\
\Phi_{2}^{C D} & =\Phi_{2}^{D D}=0 .
\end{aligned}
$$

When firms compete in terms of price in the product market in stage 2, given the technology adoption decisions of firms of stage 1, the equilibrium outcomes are as follows.

1. When no firm adopts the new technology:

$$
\begin{aligned}
p_{i}^{D D} & =\frac{a\left(2-\gamma-\gamma^{2}\right)+c(2+\gamma)}{\left(4-\gamma^{2}\right)} \\
b_{i}^{D D} & =\frac{(a-c)\left(2-\gamma-\gamma^{2}\right)}{\left(1-\gamma^{2}\right)\left(4-\gamma^{2}\right)} \\
B_{i}^{D D} & =\left(1-\gamma^{2}\right)\left[b_{i}^{D D}\right]^{2}
\end{aligned}
$$

2. When both firms adopt the new technology:

$$
\begin{aligned}
p_{1}^{C C} & =\frac{\left[\begin{array}{c}
a\left(2-\gamma-\gamma^{2}\right)+c(2+\gamma) \\
-c\left(2 k \lambda\left(y_{1}^{C C}\right)+\gamma \lambda\left(y_{2}^{C C}\right)\right)
\end{array}\right]}{\left(4-\gamma^{2}\right)} \\
b_{1}^{C C} & =\frac{\left[\begin{array}{c}
(a-c)\left(2-\gamma-\gamma^{2}\right) \\
+c\left(2 k \lambda\left(y_{1}^{C C}\right)-\gamma \lambda\left(y_{2}^{C C}\right)-\gamma^{2} k \lambda\left(y_{1}^{C C}\right)\right)
\end{array}\right]}{\left(1-\gamma^{2}\right)\left(4-\gamma^{2}\right)} \\
B_{1}^{C C} & =\left(1-\gamma^{2}\right)\left[b_{1}^{C C}\right]^{2}-\Phi_{1}^{C C} \\
p_{2}^{C C} & =\frac{\left[\begin{array}{c}
a\left(2-\gamma-\gamma^{2}\right)+c(2+\gamma) \\
-c\left(2 \lambda\left(y_{2}^{C C}\right)+\gamma k \lambda\left(y_{1}^{C C}\right)\right)
\end{array}\right]}{\left(4-\gamma^{2}\right)} \\
b_{2}^{C C} & =\frac{\left[\begin{array}{c}
(a-c)\left(2-\gamma-\gamma^{2}\right) \\
+c\left(2 \lambda\left(y_{2}^{C C}\right)-\gamma^{2} \lambda\left(y_{2}^{C C}\right)-\gamma k \lambda\left(y_{1}^{C C}\right)\right)
\end{array}\right]}{\left(1-\gamma^{2}\right)\left(4-\gamma^{2}\right)} \\
B_{2}^{C C} & =\left(1-\gamma^{2}\right)\left[b_{2}^{C C}\right]^{2}-\Phi_{2}^{C C}
\end{aligned}
$$

3. When firm 1 adopts the new technology but firm 2 does not: 


$$
\begin{aligned}
p_{1}^{C D} & =\frac{a\left(2-\gamma-\gamma^{2}\right)+c(2+\gamma)-2 c k \lambda\left(y_{1}^{C D}\right)}{\left(4-\gamma^{2}\right)} \\
b_{1}^{C D} & =\frac{(a-c)\left(2-\gamma-\gamma^{2}\right)+c k \lambda\left(y_{1}^{C D}\right)\left(2-\gamma^{2}\right)}{\left(1-\gamma^{2}\right)\left(4-\gamma^{2}\right)} \\
B_{1}^{C D} & =\left(1-\gamma^{2}\right)\left[b_{1}^{C D}\right]^{2}-\Phi_{1}^{C D} \\
p_{2}^{C D} & =\frac{a\left(2-\gamma-\gamma^{2}\right)+c(2+\gamma)-c \gamma k \lambda\left(y_{1}^{C D}\right)}{\left(4-\gamma^{2}\right)} \\
b_{2}^{C D} & =\frac{(a-c)\left(2-\gamma-\gamma^{2}\right)-c \gamma k \lambda\left(y_{1}^{C D}\right)}{\left(1-\gamma^{2}\right)\left(4-\gamma^{2}\right)} \\
B_{2}^{C D} & =\left(1-\gamma^{2}\right)\left[b_{2}^{C D}\right]^{2}
\end{aligned}
$$

4. When firm 2 adopts the new technology but firm 1 does not:

$$
\begin{aligned}
p_{1}^{D C} & =\frac{a\left(2-\gamma-\gamma^{2}\right)+c(2+\gamma)-c \gamma \lambda\left(y_{2}^{D C}\right)}{\left(4-\gamma^{2}\right)} \\
b_{1}^{D C} & =\frac{(a-c)\left(2-\gamma-\gamma^{2}\right)-c \gamma \lambda\left(y_{2}^{D C}\right)}{\left(1-\gamma^{2}\right)\left(4-\gamma^{2}\right)} \\
B_{1}^{D C} & =\left(1-\gamma^{2}\right)\left[b_{1}^{D C}\right]^{2} \\
p_{2}^{D C} & =\frac{a\left(2-\gamma-\gamma^{2}\right)+c(2+\gamma)-2 c \lambda\left(y_{2}^{D C}\right)}{\left(4-\gamma^{2}\right)} \\
b_{2}^{D C} & =\frac{(a-c)\left(2-\gamma-\gamma^{2}\right)+c \lambda\left(y_{2}^{D C}\right)\left(2-\gamma^{2}\right)}{\left(1-\gamma^{2}\right)\left(4-\gamma^{2}\right)} \\
B_{2}^{D C} & =\left(1-\gamma^{2}\right)\left[b_{2}^{D C}\right]^{2}-\Phi_{2}^{D C}
\end{aligned}
$$

\section{Equilibrium in the first-stage}

In the first stage when firm $i$ decides to choose the new technology or not, its choice is dictated by the expected value of its net payoff in the second stage. For example, if firm 1 goes for the new technology and 2 also does so, then expected net payoff to 1 is

$$
\int_{\underline{\theta}}^{\bar{\theta}} \pi_{1}^{C C}(\theta) f_{1}(\theta) d \theta=\int_{\underline{\theta}}^{\bar{\theta}}\left(\left[q_{1}^{C C}(\theta)\right]^{2}-\phi_{1}^{C C}(\theta)\right) f_{1}(\theta) d \theta
$$

Using lemma 2 we know that

$$
\begin{aligned}
& \int_{\underline{\theta}}^{\bar{\theta}} \pi_{1}^{C C}(\theta) f_{1}(\theta) d \theta=\int_{\underline{\theta}}^{\bar{\theta}}\left(\left[q_{1}^{C C}(\theta)\right]^{2}-\phi_{1}^{C C}(\theta)\right) f_{1}(\theta) d \theta \\
= & \int_{\underline{\theta}}^{\bar{\theta}}\left(\left[q_{1}^{C C}\left(x_{s}(\theta)\right)\right]^{2}-x_{s}(\theta) \theta\right) f_{2}(\theta) d \theta
\end{aligned}
$$


Let

$$
\begin{aligned}
& \hat{\pi}_{1}^{C C}=\int_{\underline{\theta}}^{\bar{\theta}}\left(\left[q_{1}^{C C}\left(x_{s}(\theta)\right)\right]^{2}-x_{s}(\theta) \theta\right) f_{2}(\theta) d \theta \\
& \hat{\pi}_{1}^{C D}=\int_{\underline{\theta}}^{\bar{\theta}}\left(\left[q_{1}^{C D}\left(x_{s}(\theta)\right)\right]^{2}-x_{s}(\theta) \theta\right) f_{2}(\theta) d \theta
\end{aligned}
$$

and so on.

Then in the first stage the choices before the two firms can be summarised as follows. The first payoff matrix deals with the case of quantity competition and the second one deals with the case of price competition.

$$
\begin{aligned}
& 1\left|\begin{array}{ccc}
C & \\
& & D \\
& & \\
C & \hat{\pi}_{1}^{C C}, \hat{\pi}_{2}^{C C} & \hat{\pi}_{1}^{C D}, \hat{\pi}_{2}^{C D} \\
D & \hat{\pi}_{1}^{D C}, \hat{\pi}_{2}^{D C} & \hat{\pi}_{1}^{D D}, \hat{\pi}_{2}^{D D} \\
& \multicolumn{2}{c}{\text { Cournot }}
\end{array}\right| \\
& \begin{array}{|ccc} 
& \multicolumn{1}{c}{2} & \\
& & D \\
C & \hat{B}_{1}^{C C}, \hat{B}_{2}^{C C} & \hat{B}_{1}^{C D}, \hat{B}_{2}^{C D} \\
D & \hat{B}_{1}^{D C}, \hat{B}_{2}^{D C} & \hat{B}_{1}^{D D}, \hat{B}_{2}^{D D} \\
& \multicolumn{2}{c}{\text { Bertrand }}
\end{array} \mid
\end{aligned}
$$

\subsection{Equilibrium when quality of the new technology lies in the interior}

We now proceed to provide our first set of major results when the quality of the new technology offered by the suppliers lies in the interior of $[0, \bar{x}]$.

Proposition 1 If $\lambda^{\prime \prime}(x)<-\frac{2 c k\left(\lambda^{\prime}(x)\right)^{2}}{(a-c)(2-\gamma)}$ for all $x \in(0, \bar{x})$ and $x_{s}(\theta) \in(0, \bar{x})$ for all $\theta \in[\underline{\theta}, \bar{\theta}]$ then the following is true.

$$
\begin{aligned}
& \hat{\pi}_{1}^{C C}>\hat{\pi}_{1}^{D C}, \hat{\pi}_{2}^{C C}>\hat{\pi}_{2}^{C D}, \hat{\pi}_{1}^{C D}>\hat{\pi}_{1}^{D D} \text { and } \hat{\pi}_{2}^{D C}>\hat{\pi}_{2}^{D D} . \\
& \hat{B}_{1}^{C C}>\hat{B}_{1}^{D C}, \hat{B}_{2}^{C C}>\hat{B}_{2}^{C D}, \hat{B}_{1}^{C D}>\hat{B}_{1}^{D D} \text { and } \hat{B}_{2}^{D C}>\hat{B}_{2}^{D D} .
\end{aligned}
$$

Proof Given in the appendix.

Proposition 2 If $\lambda^{\prime \prime}(x)<-\frac{2 c k\left(\lambda^{\prime}(x)\right)^{2}}{(a-c)(2-\gamma)}$ for all $x \in(0, \bar{x})$ and $x_{s}(\theta) \in(0, \bar{x})$ for all $\theta \in[\underline{\theta}, \bar{\theta}]$ then there is a unique subgame perfect equilibrium, where it is a strictly dominant strategy for both firms to adopt the new technology in the first stage regardless of the nature of second stage competition (Cournot or Bertrand). 
Proof The proof is straightforward and follows directly from proposition 1 and the payoff matrix provided in section 5 .

Comment We now provide a simple intuition behind our result. Given the rival's decision, if firm $i$ does not choose the new technology its marginal cost remains at $c$. Note that non-adoption of the new technology is equivalent to choosing $x=0$. If $x=0$ the second stage net profit to firm $i$ is $s(0)$ (since the firm has not chosen the new technology it does not have to pay for it). If the firm chooses the new technology it organises a scoring auction. From lemma 1 we know that a supplier of type $\theta$ will quote quality $x_{s}(\theta)=\arg \max _{x \in[0, \bar{x}]}(s(x)-x \theta)$ in the scoring auction. By hypothesis of proposition $2, x_{s}(\theta) \in(0, \bar{x})$ for all $\theta \in[\underline{\theta}, \bar{\theta}]$. This means $s\left(x_{s}(\theta)\right)-x_{s}(\theta) \theta>s(0)$ which implies that the expected value of $s\left(x_{s}(\theta)\right)-x_{s}(\theta) \theta$ is strictly greater than the expected value of $s(0)$. From lemma 2 we know that the expected payoff to firm $i$ is the expected value of $s\left(x_{s}(\theta)\right)-x_{s}(\theta) \theta$. Consequently, firm $i$ always gains by choosing the new technology.

We now compare and contrast our result to the related results in the literature. Bester and Petrakis (1993) investigate how the incentives for cost reduction in a differentiated industry depend upon the degree of product differentiation. This paper shows that Cournot competition provides a stronger incentive to innovate than Bertrand competition if the degree of product differentiation is low, and a weaker incentive if this degree is high. Pal (2010) shows that, if the given fixed cost of the technology is high, Bertrand competition provides a stronger incentive to adopt technology than Cournot competition unless the given cost-reducing effect of the technology is very low. On the contrary, if the cost of the technology is low, Cournot competition fares better than Bertrand competition in terms of technology adoption by firms. ${ }^{8}$

In sharp contrast to the related results in the literature we show that the nature of competition (Cournot or Bertrand) has no effect on the equilibrium decision of the firms to adopt the new technology when the quality of the new technology offered by the suppliers lies in the interior of $[0, \bar{x}]$. In our model both firms chooses to adopt the new technology in the first stage regardless of the value of $\gamma$ in the relevant range and regardless of the nature of second stage competition (Cournot or Bertrand).

\subsection{Equilibrium when quality of the new technology is $\bar{x}$}

The previous section dealt with the case where the quality of the new technology offered by the suppliers lies in the interior of $[0, \bar{x}]$. Now we attempt to analyse the case when there is a corner solution (where the suppliers offer quality $\bar{x}$ ). We show that it is possible to have an equilibrium outcome like where either (i) none of the firms adopts the new technology or (ii) where only one of the firms adopts the new technology. We now provide our next result.

Lemma 3 If $\lambda^{\prime \prime}(x) \geq 0$ for all $x \in(0, \bar{x})$ then $x_{s}(\theta)=\bar{x}$ for all $\theta \in[\underline{\theta}, \bar{\theta}]$. This is true regardless of the nature of second stage competition (Cournot or Bertrand).

\footnotetext{
${ }^{8}$ In Elberfeld and Nti (2004) the second stage competition is Cournot only and consequently, the issues regarding differences due to nature of competition (Cournot or Bertrand) does not arise. In Das-Verma (2003) and Moldovanu and Sela (2003) there is a reduction only in the winning bidder's cost in the product market. In our set-up any firm can choose to reduce its marginal cost by purchasing the new technology in a scoring auction. The new technology is not protected by any patent. Hence, in our model it is possible to have a scenario where both firms reduce marginal costs by adopting the new technology.
} 
Proof Given in the appendix.

In this case (when $\lambda^{\prime \prime}(x) \geq 0$ ), any supplier of the new technology, regardless of its type, offer $\bar{x}$ in equilibrium. In the scoring auction equilibrium we have the following:

$$
\begin{aligned}
x_{s}(\theta) & =\bar{x} \text { for all } \theta \in[\underline{\theta}, \bar{\theta}] . \\
\phi_{s}(\theta) & =\bar{x}\left[\theta+\int_{\theta}^{\bar{\theta}}\left(\frac{1-F(t)}{1-F(\theta)}\right)^{n-1} d t\right]
\end{aligned}
$$

This means

$$
\begin{aligned}
& x_{1}^{C C}=x_{1}^{C D}=x_{2}^{C C}=x_{2}^{D C}=\bar{x} \\
& y_{1}^{C C}=y_{1}^{C D}=y_{2}^{C C}=y_{2}^{D C}=\bar{x}
\end{aligned}
$$

And as before

$$
\begin{aligned}
x_{1}^{D C} & =x_{1}^{D D}=x_{2}^{C D}=x_{2}^{D D}=0 \\
\phi_{1}^{D C} & =\phi_{1}^{D D}=\phi_{2}^{C D}=\phi_{2}^{D D}=0 \\
y_{1}^{D C} & =y_{1}^{D D}=y_{2}^{C D}=y_{2}^{D D}=0 \\
\Phi_{1}^{D C} & =\Phi_{1}^{D D}=\Phi_{2}^{C D}=\Phi_{2}^{D D}=0
\end{aligned}
$$

Let

$$
z=\int_{\underline{\theta}}^{\bar{\theta}} \theta f_{2}(\theta) d \theta
$$

That is, $z$ is the expected value of the second lowest type.

Now note the following:

$$
\begin{aligned}
\hat{\pi}_{1}^{C C} & =\int_{\underline{\theta}}^{\bar{\theta}}\left(\left[q_{1}^{C C}\left(x_{s}(\theta)\right)\right]^{2}-x_{s}(\theta) \theta\right) f_{2}(\theta) d \theta \\
& =\frac{1}{\left(4-\gamma^{2}\right)} \int_{\underline{\theta}}^{\bar{\theta}}\left[((a-c)(2-\gamma)+c \lambda(\bar{x})\{2 k-\gamma\})^{2}-\bar{x} \theta\right] f_{2}(\theta) d \theta \\
& =\frac{((a-c)(2-\gamma)+c \lambda(\bar{x})\{2 k-\gamma\})^{2}}{\left(4-\gamma^{2}\right)}-\bar{x} z .
\end{aligned}
$$

Similarly we can compute $\hat{\pi}_{1}^{D C}, \hat{\pi}_{2}^{C C}, \hat{\pi}_{2}^{C D}, \hat{\pi}_{1}^{C D}, \hat{\pi}_{1}^{D D}, \hat{\pi}_{2}^{D C}$ and $\hat{\pi}_{2}^{D D}$. Also we can compute $\hat{B}_{1}^{C C}, \hat{B}_{1}^{D C}, \hat{B}_{2}^{C C}, \hat{B}_{2}^{C D}, \hat{B}_{1}^{C D}, \hat{B}_{1}^{D D}, \hat{B}_{2}^{D C}$ and $\hat{B}_{2}^{D D}$.

We now come to our next result. 
Lemma 4 (i) $\exists$ unique $\underline{k} \in(1, \infty)$ s.t.

$$
(a-c)(2-\gamma)(\underline{k}-1)+c \lambda(\bar{x})\left(\underline{k}^{2}-\underline{k} \gamma-1\right)=0 .
$$

(ii) $\exists$ unique $\underline{\kappa} \in(1, \infty)$ s.t.

$$
2(a-c)\left(2-\gamma-\gamma^{2}\right)(\underline{\kappa}-1)+c \lambda(\bar{x})\left[\left(2-\gamma^{2}\right)\left(\underline{\kappa}^{2}-1\right)-2 \underline{\kappa} \gamma\right]=0 .
$$

Proof Given in the appendix.

remark 2 For the rest of the paper we will assume that $\lambda^{\prime \prime}(x) \geq 0$ for all $x \in(0, \bar{x})$. From lemma 3 we know that this means any supplier of the new technology, regardless of its type, offers $\bar{x}$ in equilibrium. This implies that when a firm chooses to adopt the new technology, it knows with certainty both the quality $(\bar{x})$ of the new technology and also the associated decrease in marginal cost of production. This aspect is very different from our previous case where the quality offered by the suppliers lies in the interior of $[0, \bar{x}]$. However, as in the previous case, before the outcome of the scoring auction is known, the firms do not know with certainty the cost of the new technology.

Now note that following results.

Lemma 5 (i) If $k \in(1, \underline{k})$ then

$$
\hat{\pi}_{1}^{C D}-\hat{\pi}_{1}^{D D}>\hat{\pi}_{2}^{D C}-\hat{\pi}_{2}^{D D}>\hat{\pi}_{1}^{C C}-\hat{\pi}_{1}^{D C}>\hat{\pi}_{2}^{C C}-\hat{\pi}_{2}^{C D} .
$$

(ii) If $k \in(\underline{k}, \infty)$ then

$$
\hat{\pi}_{1}^{C D}-\hat{\pi}_{1}^{D D}>\hat{\pi}_{1}^{C C}-\hat{\pi}_{1}^{D C}>\hat{\pi}_{2}^{D C}-\hat{\pi}_{2}^{D D}>\hat{\pi}_{2}^{C C}-\hat{\pi}_{2}^{C D} .
$$

(iii) If $k \in(1, \underline{\kappa})$ then

$$
\hat{B}_{1}^{C D}-\hat{B}_{1}^{D D}>\hat{B}_{2}^{D C}-\hat{B}_{2}^{D D}>\hat{B}_{1}^{C C}-\hat{B}_{1}^{D C}>\hat{B}_{2}^{C C}-\hat{B}_{2}^{C D} .
$$

(iv) If $k \in(\underline{\kappa}, \infty)$ then

$$
\hat{B}_{1}^{C D}-\hat{B}_{1}^{D D}>\hat{B}_{1}^{C C}-\hat{B}_{1}^{D C}>\hat{B}_{2}^{D C}-\hat{B}_{2}^{D D}>\hat{B}_{2}^{C C}-\hat{B}_{2}^{C D} .
$$

Proof Given in the appendix

Now note that

$$
\begin{gathered}
\hat{\pi}_{2}^{C C}-\hat{\pi}_{2}^{C D} \geq 0 \Longleftrightarrow \frac{4 c \lambda(\bar{x})}{\left(4-\gamma^{2}\right)^{2}}[(a-c)(2-\gamma)+c \lambda(\bar{x})(1-k \gamma)] \geq \bar{x} z \\
\hat{B}_{2}^{C C}-\hat{B}_{2}^{C D} \geq 0 \Longleftrightarrow \frac{c \lambda(\bar{x})\left(2-\gamma^{2}\right)}{\left(1-\gamma^{2}\right)\left(4-\gamma^{2}\right)^{2}}\left[2(a-c)\left(2-\gamma-\gamma^{2}\right)+c \lambda(\bar{x})\left(2-\gamma^{2}-2 k \gamma\right)\right] \geq \bar{x} z \\
\hat{\pi}_{1}^{C D}-\hat{\pi}_{1}^{D D} \leq 0 \Longleftrightarrow \frac{4 c k \lambda(\bar{x})}{\left(4-\gamma^{2}\right)^{2}}[(a-c)(2-\gamma)+c k \lambda(\bar{x})] \leq \bar{x} z \\
\hat{B}_{1}^{C D}-\hat{B}_{1}^{D D} \leq 0 \Longleftrightarrow \frac{c k \lambda(\bar{x})\left(2-\gamma^{2}\right)}{\left(1-\gamma^{2}\right)\left(4-\gamma^{2}\right)^{2}}\left[2(a-c)\left(2-\gamma-\gamma^{2}\right)+c k \lambda(\bar{x})\left(2-\gamma^{2}\right)\right] \leq \bar{x} z
\end{gathered}
$$

The next two results provide conditions under which either $(C, C)$ is the equilibrium outcome or $(D, D)$ is the equilibrium outcome. 
Proposition 3 (i) If

$$
\frac{4 c \lambda(\bar{x})}{\left(4-\gamma^{2}\right)^{2}}[(a-c)(2-\gamma)+c \lambda(\bar{x})(1-k \gamma)] \geq \bar{x} z
$$

then both firms adopting the new technology is the unique subgame perfect equilibrium when firms engage in quantity competition in the second stage. That is, the equilibrium outcome in the first stage is $(C, C)$.

(ii) If

$$
\frac{c \lambda(\bar{x})\left(2-\gamma^{2}\right)}{\left(1-\gamma^{2}\right)\left(4-\gamma^{2}\right)^{2}}\left[2(a-c)\left(2-\gamma-\gamma^{2}\right)+c \lambda(\bar{x})\left(2-\gamma^{2}-2 k \gamma\right)\right] \geq \bar{x} z
$$

then both firms adopting the new technology is the unique subgame perfect equilibrium when firms engage in price competition in the second stage. That is, the equilibrium outcome in the first stage is $(C, C)$.

Proof Given in the appendix.

Comment From Proposition 3 it follows that for any given $\gamma \in(0, \gamma)$ and $c$, when $a$ is large enough then $(C, C)$ is always the unique equilibrium outcome for both quantity and price competition. The intuition behind this is simple. $a$ can be thought of as proxy for market size. When market size is large enough any firm has an incentive to lower marginal cost and, thereby increasing it's market share as tapping the large market fetches more gains as compared to the additional cost of purchasing the new technology.

We now show that is also possible to have equilibria where no firm chooses to adopt the new technology.

Proposition 4 (i) If

$$
\frac{4 c k \lambda(\bar{x})}{\left(4-\gamma^{2}\right)^{2}}[(a-c)(2-\gamma)+c k \lambda(\bar{x})] \leq \bar{x} z
$$

then both firms not adopting the new technology is the unique subgame perfect equilibrium when firms engage in quantity competition in the second stage. That is, the equilibrium outcome in the first stage is $(D, D)$.

(ii) If

$$
\frac{c k \lambda(\bar{x})\left(2-\gamma^{2}\right)}{\left(1-\gamma^{2}\right)\left(4-\gamma^{2}\right)^{2}}\left[2(a-c)\left(2-\gamma-\gamma^{2}\right)+c k \lambda(\bar{x})\left(2-\gamma^{2}\right)\right] \leq \bar{x} z
$$

then both firms not adopting the new technology is the unique subgame perfect equilibrium when firms engage in price competition in the second stage. That is, the equilibrium outcome in the first stage is $(D, D)$.

Proof Given in the appendix. 
Comment Proposition 4 shows that for any given $\gamma \in(0, \underline{\gamma})$ and any $a$, if $c$ is small enough then $(D, D)$ is always the unique equilibrium outcome for both quantity and price competition. The intuition behind this is simple. When $c$ is very small, then even without adopting the new technology, a firm's costs are low and hence the incentive to lower it further is not there as it has to incur an additional fixed cost (of purchasing it).

We now show that in both quantity and price competition, it is also possible to have equilibria where one firm chooses to adopt the new technology whereas the other firm does not.

\subsubsection{Quantity competition}

We first deal with Cournot competition. First, consider the following four terms: $\left(\hat{\pi}_{1}^{C D}-\hat{\pi}_{1}^{D D}\right)$, $\left(\hat{\pi}_{2}^{D C}-\hat{\pi}_{2}^{D D}\right),\left(\hat{\pi}_{1}^{C C}-\hat{\pi}_{1}^{D C}\right)$, and $\left(\hat{\pi}_{2}^{C C}-\hat{\pi}_{2}^{C D}\right)$. Lemma 5 show that regardless of the value of $k$, the term $\left(\hat{\pi}_{1}^{C D}-\hat{\pi}_{1}^{D D}\right)$ is strictly higher than the others and the term $\left(\hat{\pi}_{2}^{C C}-\hat{\pi}_{2}^{C D}\right)$ is the strictly lower than the others. The relative position of $\left(\hat{\pi}_{2}^{D C}-\hat{\pi}_{2}^{D D}\right)$ and $\left(\hat{\pi}_{1}^{C C}-\hat{\pi}_{1}^{D C}\right)$ depends on whether $k$ is higher than $\underline{k}$ or not.

We now provide the main results with quantity competition and corner solution in quality. First note that when $k \in(1, \underline{k})$ then $\hat{\pi}_{1}^{C D}-\hat{\pi}_{1}^{D D}>\hat{\pi}_{2}^{D C}-\hat{\pi}_{2}^{D D}>\hat{\pi}_{1}^{C C}-\hat{\pi}_{1}^{D C}>\hat{\pi}_{2}^{C C}-\hat{\pi}_{2}^{C D}$.

Proposition 5 Suppose $k \in(1, \underline{k})$ and there is quantity competition in the second stage.

(i) If either

$$
\hat{\pi}_{1}^{C D}-\hat{\pi}_{1}^{D D}>0>\hat{\pi}_{2}^{D C}-\hat{\pi}_{2}^{D D}>\hat{\pi}_{1}^{C C}-\hat{\pi}_{1}^{D C}>\hat{\pi}_{2}^{C C}-\hat{\pi}_{2}^{C D}
$$

or

$$
\hat{\pi}_{1}^{C D}-\hat{\pi}_{1}^{D D}>\hat{\pi}_{2}^{D C}-\hat{\pi}_{2}^{D D}>\hat{\pi}_{1}^{C C}-\hat{\pi}_{1}^{D C}>0>\hat{\pi}_{2}^{C C}-\hat{\pi}_{2}^{C D}
$$

then there is a unique subgame perfect equilibrium where firm 1 chooses to adopt but firm 2 does not. That is, the equilibrium outcome in the first stage is $(C, D)$.

(ii) If

$$
\hat{\pi}_{1}^{C D}-\hat{\pi}_{1}^{D D}>\hat{\pi}_{2}^{D C}-\hat{\pi}_{2}^{D D}>0>\hat{\pi}_{1}^{C C}-\hat{\pi}_{1}^{D C}>\hat{\pi}_{2}^{C C}-\hat{\pi}_{2}^{C D}
$$

then there are two subgame perfect equilibria where one firm chooses to adopt but the other does not. That is, the equilibrium outcome in the first stage is either $(C, D)$ or $(D, C)$.

Proof The proof follows directly from the payoff matrix provided in section 5 .

Note that lemma 5 also shows that when $k \in(\underline{k}, \infty)$ then $\hat{\pi}_{1}^{C D}-\hat{\pi}_{1}^{D D}>\hat{\pi}_{1}^{C C}-\hat{\pi}_{1}^{D C}>$ $\hat{\pi}_{2}^{D C}-\hat{\pi}_{2}^{D D}>\hat{\pi}_{2}^{C C}-\hat{\pi}_{2}^{C D}$.

Proposition 6 Suppose $k \in(\underline{k}, \infty)$ and there is quantity competition in the second stage.

(i) If either

$$
\hat{\pi}_{1}^{C D}-\hat{\pi}_{1}^{D D}>0>\hat{\pi}_{1}^{C C}-\hat{\pi}_{1}^{D C}>\hat{\pi}_{2}^{D C}-\hat{\pi}_{2}^{D D}>\hat{\pi}_{2}^{C C}-\hat{\pi}_{2}^{C D}
$$

or

$$
\hat{\pi}_{1}^{C D}-\hat{\pi}_{1}^{D D}>\hat{\pi}_{1}^{C C}-\hat{\pi}_{1}^{D C}>0>\hat{\pi}_{2}^{D C}-\hat{\pi}_{2}^{D D}>\hat{\pi}_{2}^{C C}-\hat{\pi}_{2}^{C D}
$$

or

$$
\hat{\pi}_{1}^{C D}-\hat{\pi}_{1}^{D D}>\hat{\pi}_{1}^{C C}-\hat{\pi}_{1}^{D C}>\hat{\pi}_{2}^{D C}-\hat{\pi}_{2}^{D D}>0>\hat{\pi}_{2}^{C C}-\hat{\pi}_{2}^{C D}
$$

then there is a unique subgame perfect equilibrium where firm 1 chooses to adopt but firm 2 does not. That is, the equilibrium outcome in the first stage is $(C, D)$. 
Proof The proof follows directly from the payoff matrix provided in section 5 .

\subsubsection{Bertrand Competition}

We next consider price competition. The results are very similar. The proofs of propositions 7 and 8 follow directly from the payoff matrix provided in section 5 .

As before consider the following four terms: $\left(\hat{B}_{1}^{C D}-\hat{B}_{1}^{D D}\right),\left(\hat{B}_{2}^{D C}-\hat{B}_{2}^{D D}\right),\left(\hat{B}_{1}^{C C}-\hat{B}_{1}^{D C}\right)$, and $\left(\hat{B}_{2}^{C C}-\hat{B}_{2}^{C D}\right)$. Lemma 5 show that regardless of the value of $k$, the term $\left(\hat{B}_{1}^{C D}-\hat{B}_{1}^{D D}\right)$ is strictly higher than the others and the term $\left(\hat{B}_{2}^{C C}-\hat{B}_{2}^{C D}\right)$ is the strictly lower than the others. The relative position of $\left(\hat{B}_{2}^{D C}-\hat{B}_{2}^{D D}\right)$ and $\left(\hat{B}_{1}^{C C}-\hat{B}_{1}^{D C}\right)$ depends on whether $k$ is higher than $\underline{\kappa}$, or not.

When $k \in(1, \underline{\kappa}$,$) then \hat{B}_{1}^{C D}-\hat{B}_{1}^{D D}>\hat{B}_{2}^{D C}-\hat{B}_{2}^{D D}>\hat{B}_{1}^{C C}-\hat{B}_{1}^{D C}>\hat{B}_{2}^{C C}-\hat{B}_{2}^{C D}$.

Proposition 7 Suppose $k \in(1, \underline{\kappa})$ and there is price competition in the second stage.

(i) If either

$$
\hat{B}_{1}^{C D}-\hat{B}_{1}^{D D}>0>\hat{B}_{2}^{D C}-\hat{B}_{2}^{D D}>\hat{B}_{1}^{C C}-\hat{B}_{1}^{D C}>\hat{B}_{2}^{C C}-\hat{B}_{2}^{C D}
$$

or

$$
\hat{B}_{1}^{C D}-\hat{B}_{1}^{D D}>\hat{B}_{2}^{D C}-\hat{B}_{2}^{D D}>\hat{B}_{1}^{C C}-\hat{B}_{1}^{D C}>0>\hat{B}_{2}^{C C}-\hat{B}_{2}^{C D}
$$

then there is a unique subgame perfect equilibrium where firm 1 chooses to adopt but firm 2 does not. That is, the equilibrium outcome in the first stage is $(C, D)$.

(ii) If

$$
\hat{B}_{1}^{C D}-\hat{B}_{1}^{D D}>\hat{B}_{2}^{D C}-\hat{B}_{2}^{D D}>0>\hat{B}_{1}^{C C}-\hat{B}_{1}^{D C}>\hat{B}_{2}^{C C}-\hat{B}_{2}^{C D}
$$

then there are two subgame perfect equilibria where one firm chooses to adopt but the other does not. That is, the equilibrium outcome in the first stage is either $(C, D)$ or $(D, C)$.

Lemma 5 also shows that when $k \in(\underline{\kappa}, \infty)$ then $\hat{B}_{1}^{C D}-\hat{B}_{1}^{D D}>\hat{B}_{1}^{C C}-\hat{B}_{1}^{D C}>\hat{B}_{2}^{D C}-\hat{B}_{2}^{D D}>$ $\hat{B}_{2}^{C C}-\hat{B}_{2}^{C D}$.

Proposition 8 Suppose $k \in(\underline{\kappa}, \infty)$ and there is price competition in the second stage.

(i) If either

$$
\hat{B}_{1}^{C D}-\hat{B}_{1}^{D D}>0>\hat{B}_{1}^{C C}-\hat{B}_{1}^{D C}>\hat{B}_{2}^{D C}-\hat{B}_{2}^{D D}>\hat{B}_{2}^{C C}-\hat{B}_{2}^{C D}
$$

or

$$
\hat{B}_{1}^{C D}-\hat{B}_{1}^{D D}>\hat{B}_{1}^{C C}-\hat{B}_{1}^{D C}>0>\hat{B}_{2}^{D C}-\hat{B}_{2}^{D D}>\hat{B}_{2}^{C C}-\hat{B}_{2}^{C D}
$$

or

$$
\hat{B}_{1}^{C D}-\hat{B}_{1}^{D D}>\hat{B}_{1}^{C C}-\hat{B}_{1}^{D C}>\hat{B}_{2}^{D C}-\hat{B}_{2}^{D D}>0>\hat{B}_{2}^{C C}-\hat{B}_{2}^{C D}
$$

then there is a unique subgame perfect equilibrium where firm 1 chooses to adopt but firm 2 does not. That is, the equilibrium outcome in the first stage is $(C, D)$. 
Comment Note that $k$ represents firm 1's relative competence vis-a-vis firm 2. Since $k \geq 1$ firm 1 is more competent than firm 2. Hence, when $k$ is high enough (i.e. either $k>\underline{k}$ in quantity competition or $k>\underline{\kappa}$ in price competition), then it is never an equilibrium outcome where firm 1 does not adopt the new technology but firm 2 does. This arises because firm 1 is far more adept than firm 2 at reducing marginal cost. When $k$ is low enough (i.e. $k \in(1, \underline{k})$ in quantity competition or $k \in(1, \underline{\kappa})$ in price competition) and if it's the case that the outcome $(D, D)$ does not arise in equilibrium, then for all feasible parameter values there is always an equilibrium where firm 1 adopts the new technology in equilibrium.

\subsubsection{Cournot vs Bertrand}

We proceed to explore the question as to whether the nature of competition (Cournot or Bertrand) make a difference. We show that the nature of competition sometimes make a difference in the case when there is a corner solution (where the suppliers offer quality $\bar{x}$ ).

In Cournot competition, a reduction in firm $i$ 's unit cost increases its output, $q_{i}$, and decreases it rivals output, $q_{j}$. Total supply, $q_{i}+q_{j}$, is increased. This particular effect is strategically advantageous for firm $i$. On the other hand, in Bertrand competition, a reduction in firm $i$ 's unit cost decreases price of both firms. This is strategically disadvantageous for firm $i$ because its output is positively related to the other firm's price. This implies that in contrast with Cournot competition, Bertrand competition creates a negative strategic incentive to adopt a new technology. However, it may be noted that the gains from a cost reduction not only depend on the strategic effect but also on how much total production costs are decreased. Therefore, if price competition results in a higher output than quantity competition, it may be the case that Bertrand competition induces a greater overall incentive to adopt a new technology than Cournot competition. ${ }^{9}$

We now come to our next result.

Lemma 6 If $\frac{2 a-2 c}{2 a-2 c+c k \lambda(\bar{x})}<\underline{\gamma}$, then for $\gamma \in\left(0, \frac{2 a-2 c}{2 a-2 c+c k \lambda(\bar{x})}\right)$

$$
\hat{\pi}_{1}^{C D}-\hat{\pi}_{1}^{D D}>\hat{B}_{1}^{C D}-\hat{B}_{1}^{D D}
$$

and for $\gamma \in\left(\frac{2 a-2 c}{2 a-2 c+c k \lambda(\bar{x})}, \underline{\gamma}\right)$

$$
\hat{\pi}_{1}^{C D}-\hat{\pi}_{1}^{D D}<\hat{B}_{1}^{C D}-\hat{B}_{1}^{D D}
$$

Proof Given in the appendix.

Now from lemma 6 we know that the following scenarios are possible: (i) $\gamma \in\left(0, \frac{2 a-2 c}{2 a-2 c+c k \lambda(\bar{x})}\right)$ and $\hat{\pi}_{1}^{C D}-\hat{\pi}_{1}^{D D}>0>\hat{B}_{1}^{C D}-\hat{B}_{1}^{D D}$ and (ii) $\gamma \in\left(\frac{2 a-2 c}{2 a-2 c+c k \lambda(\bar{x})}, \underline{\gamma}\right)$ and $\hat{\pi}_{1}^{C D}-\hat{\pi}_{1}^{D D}<0<\hat{B}_{1}^{C D}-\hat{B}_{1}^{D D}$.

This brings us to our next main result. The proof follows directly from the payoff matrix provided in section 5 .

Proposition 9 (i) If $\gamma \in\left(0, \frac{2 a-2 c}{2 a-2 c+c k \lambda(\bar{x})}\right)$ and $\hat{\pi}_{1}^{C D}-\hat{\pi}_{1}^{D D}>0>\hat{B}_{1}^{C D}-\hat{B}_{1}^{D D}$ then $(D, D)$ is the unique equilibrium outcome under Bertrand competition. However, $(D, D)$ is never an equilibrium under Cournot competition. (ii) If $\gamma \in\left(\frac{2 a-2 c}{2 a-2 c+c k \lambda(\bar{x})}, \underline{\gamma}\right)$ and $\hat{\pi}_{1}^{C D}-\hat{\pi}_{1}^{D D}<0<\hat{B}_{1}^{C D}-\hat{B}_{1}^{D D}$ then $(D, D)$ is the unique equilibrium outcome under Cournot competition. However, $(D, D)$ is never an equilibrium under Bertrand competition.

\footnotetext{
${ }^{9}$ We follow section 4 of Bester and Petrakis (1993) for the discussion in this paragraph.
} 
Comment Proposition 9 shows that when the degree of substitutability $(\gamma)$ is low then $(D, D)$ is the only equilibrium under Bertrand competition whereas it is never an equilibrium under Cournot competition. Also, when the degree of substitutability $(\gamma)$ is high then $(D, D)$ is the only equilibrium under Cournot competition whereas it is never an equilibrium under Bertrand. This conclusion is somewhat similar to Bester and Petrakis (1993) where it is shown that Cournot competition provides a stronger incentive to innovate than Bertrand competition if the degree of substitutability $(\gamma)$ is low, and a weaker incentive if this degree is high.

The intuition behind this result is related to the differences in the strategic effects under Cournot and Bertrand competition. ${ }^{10}$ Note that when $\gamma=0$, price and quantity decisions result in the same outcome. For low values of $\gamma$ the type of market competition has only a small impact on firm $i$ 's output. This implies that the gain from reducing total cost does not differ very much in the two categories of equilibrium (Bertrand or Cournot) when $\gamma$ is low. Consequently, the strategic effect dominates and new technology adoption becomes relatively more attractive under Cournot competition.

The following may now be noted.

Lemma 7 The following inequality holds true for any $k \geq 1$ and for any $\gamma \in(0, \underline{\gamma})$.

$$
\hat{\pi}_{2}^{C C}-\hat{\pi}_{2}^{C D}>\hat{B}_{2}^{C C}-\hat{B}_{2}^{C D}
$$

Proof Given in the appendix.

From lemma 7 it is clear that the following is a possibility.

$$
\hat{\pi}_{2}^{C C}-\hat{\pi}_{2}^{C D}>0>\hat{B}_{2}^{C C}-\hat{B}_{2}^{C D}
$$

The above is equivalent to

$$
\begin{aligned}
& \frac{4 c \lambda(\bar{x})}{\left(4-\gamma^{2}\right)^{2}}[(a-c)(2-\gamma)+c \lambda(\bar{x})(1-k \gamma)] \\
> & \bar{x} z>\frac{c \lambda(\bar{x})\left(2-\gamma^{2}\right)}{\left(1-\gamma^{2}\right)\left(4-\gamma^{2}\right)^{2}}\left[2(a-c)\left(2-\gamma-\gamma^{2}\right)+c \lambda(\bar{x})\left(2-\gamma^{2}-2 k \gamma\right)\right]
\end{aligned}
$$

We now come to our last main result. The proof follows directly from the payoff matrix provided in section 5 .

Proposition 10 If

$$
\begin{aligned}
& \frac{4 c \lambda(\bar{x})}{\left(4-\gamma^{2}\right)^{2}}[(a-c)(2-\gamma)+c \lambda(\bar{x})(1-k \gamma)] \\
> & \bar{x} z>\frac{c \lambda(\bar{x})\left(2-\gamma^{2}\right)}{\left(1-\gamma^{2}\right)\left(4-\gamma^{2}\right)^{2}}\left[2(a-c)\left(2-\gamma-\gamma^{2}\right)+c \lambda(\bar{x})\left(2-\gamma^{2}-2 k \gamma\right)\right]
\end{aligned}
$$

then under Cournot competition $(C, C)$ is the unique equilibrium outcome. Under Bertrand competition at most one firm chooses to adopt the new technology in equilibrium.

\footnotetext{
${ }^{10}$ We follow section 5 of Bester and Petrakis (1993) for this intuition.
} 
Comment From lemma 5 we know that the term $\left(\hat{\pi}_{2}^{C C}-\hat{\pi}_{2}^{C D}\right)$ is the lowest among the four terms: $\left(\hat{\pi}_{1}^{C D}-\hat{\pi}_{1}^{D D}\right),\left(\hat{\pi}_{2}^{D C}-\hat{\pi}_{2}^{D D}\right),\left(\hat{\pi}_{1}^{C C}-\hat{\pi}_{1}^{D C}\right)$ and $\left(\hat{\pi}_{2}^{C C}-\hat{\pi}_{2}^{C D}\right)$. As noted before, we have

$$
\hat{\pi}_{2}^{C C}-\hat{\pi}_{2}^{C D}>0 \Leftrightarrow \bar{x} z<\frac{4 c \lambda(\bar{x})}{\left(4-\gamma^{2}\right)^{2}}[(a-c)(2-\gamma)+c \lambda(\bar{x})(1-k \gamma)]
$$

Since $\bar{x} z$ is the expected cost of acquiring the new technology it means that when $\left(\hat{\pi}_{2}^{C C}-\hat{\pi}_{2}^{C D}\right)>0$ then the cost of the new technology is relatively low. In proposition 10 we get that under Cournot competition $(C, C)$ is the unique equilibrium outcome. But under Bertrand competition at most one firm chooses to adopt the new technology in equilibrium. That is, the incentive to invest in a new technology is higher under Cournot when its cost $(\bar{x} z)$ is relatively low. This is somewhat similar to Pal (2010).

A simple intuition behind our result is as follows. ${ }^{11}$ Following technology adoption, Bertrand competition not only leads to lower prices (strategic effect), but also results in a lower market share of the non-adopting firm (selection effect) than Cournot competition. We have noted earlier that the strategic effect provides more incentive to adopt technology under Cournot competition than under Bertrand competition. However, the selection effect works in the opposite direction.

Note that when only one firm adopts the cost-reducing technology, the selection effect dominates the strategic effect and as a result new technology adoption is more attractive under Bertrand competition than under Cournot competition. On the other hand, if both firms adopt the technology, the selection effect disappears and the gain from technology adoption are higher under Cournot competition than under Bertrand competition. A firm's gain from technology adoption is clearly higher when only that particular firm adopts the technology. Therefore, when the cost of the technology is relatively low, in equilibrium, both firms adopt the technology under Cournot competition whereas it's not so under Bertrand competition.

\section{Conclusion}

This paper analyses the incentives to adopt cost-reducing technology by firms in a horizontally differentiated industry under two alternative categories of product market competition, Cournot and Bertrand. Our approach is different from the related papers in the literature. We consider a two-stage game. In the first stage firms simultaneously and independently decide whether or not to adopt the new technology. In the second stage the firms engage either in Cournot competition or in Bertrand competition in a differentiated product market. In our model there are several suppliers of a new technology. The quality of the new technology lies in the interval $[0, \bar{x}]$. Higher is the quality, the lower will be the marginal cost. The extent of the cost reduction depends on the quality of the new technology. A firm has to buy the technology in a 'scoring auction'. This means that both the cost of buying this new technology and the quality of the technology (which affects the marginal cost of production) are no longer given but they depend on the equilibrium outcome in the scoring auction.

In contrast to results in the literature we show the following:

1. The nature of competition (Cournot or Bertrand) has no effect on the equilibrium decision of the firms to adopt the new technology when the quality of the new technology offered by the suppliers lies in the interior of $[0, \bar{x}]$. In this case, both firms adopt new technology in equilibrium.

\footnotetext{
${ }^{11}$ We follow Pal (2010) to provide our intuition.
} 
2. However, when the quality of the new technology offered by the suppliers is $\bar{x}$ for all types (corner solution), then it is possible to have an equilibrium where only one firm (or no firm) adopts the new technology.

3. When there is corner solution in quality, in some cases the nature of competition (Cournot or Bertrand) makes a difference in the equilibrium outcomes. This depends on the degree of product differentiation and the expected cost of acquiring the new technology. 


\section{References}

1. Bester, H. and E. Petrakis (1993) "The incentives for cost reduction in a differentiated industry," International Journal of Industrial Organization, vol. 11, pp. 519- 534.

2. Bonnano, G., Haworth, B. (1998) "Intensity of competition and the choice between product and process innovation," International Journal of Industrial Organization, vol. 16, pp. 495510 .

3. Boone, J. (2001) "Intensity of competition and the incentive to innovate," International Journal of Industrial Organization, vol. 19, pp. 705- 726.

4. Branco, F. (1997) "The design of multidimensional auctions," Rand Journal of Economics, vol. 28 , pp. $63-81$.

5. Che, Y-K (1993) "Design competition through multidimensional auctions," Rand Journal of Economics, vol. 24, pp. 668-680.

6. Delbono, F. and V. Denicolo (1990) "R\&D investment in a symmetric and homogeneous oligopoly," International Journal of Industrial Organization, vol. 8, pp. 297-313.

7. Das Varma, G. (2003) "Bidding for a process innovation under alternative modes of competition," International Journal of Industrial Organization, vol. 21, pp. 15-37.

8. Dasgupta, P. and J. Stiglitz (1980) "Uncertainty, industrial structure, and the speed of R\&D," Bell Journal of Economics, vol. 11, pp. 1-28.

9. Dixit, A. (1979) "A Model of Duopoly Suggesting a Theory of Entry Barriers," The Bell Journal of Economics, vol. 10, pp. 20-32.

10. Elberfeld, W. and K.O. Nti (2004) "Oligopolistic competition and new technology adoption under uncertainty," Journal of Economics, vol. 82, pp. 105- 121.

11. Hackner, J. (2000) "A Note on Price and Quantity Competition in Differentiated Oligopolies," Journal of Economic Theory, vol. 93, pp. 233-239.

12. Jensen, R. (1992) "Innovation adoption and welfare under uncertainty," Journal of Industrial Economics, vol. 40, pp. 173-180.

13. Keller, G. (2011) "Airbus says IndiGo buying 180 aircraft," Seattle Times, January 11, 2011. $<<$ http://seattletimes.com/html/businesstechnology/2013907245_apeufranceairbus.html >>

14. Krishna, V. (2010) Auction Theory (2nd Edition), Academic Press, San Diego, California, U.S.A.

15. Loury, G. (1979) "Market Structure and Innovation," Quarterly Journal of Economics, vol. 93, pp. 395-410.

16. Moldovanu, B. and A. Sela (2003) "Patent licensing to Bertrand competitors," International Journal of Industrial Organization, vol. 21, pp. 1-13. 
17. Pal, R. (2010) "Technology adoption in a differentiated duopoly: Cournot vs Bertrand," Research in Economics, vol. 64, pp. 128-136

18. Singh, N. and X. Vives (1984) "Price and Quantity Competition in a Differentiated Duopoly," Rand Journal of Economics, vol. 15, pp. 546- 554.

19. Singhal, M. (2011) "Biggest order: IndiGo airline picks up 180 jets," Economic Times, January 13, $2011<<$ http://articles.economictimes.indiatimes.com/2011-01-13/news/28428535_1_airbusaircraft-rahul-bhatia-airbus-a320s $>>$

20. Zanchettin, P. (2006) "Differentiated duopoly with asymmetric costs," Journal of Economics and Management Strategy, vol. 15, pp. 999- 1015. 


\section{Appendix}

Proof of Lemma 2 We have to show that

$$
\begin{aligned}
& \int_{\underline{\theta}}^{\bar{\theta}}\left(s\left(x_{s}(\theta)\right)-x_{s}(\theta) \theta-\int_{\theta}^{\bar{\theta}} x_{s}(t)\left[\frac{1-F(t)}{1-F(\theta)}\right]^{n-1}\right) f_{1}(\theta) d \theta \\
= & \int_{\underline{\theta}}^{\bar{\theta}}\left[s\left(x_{s}(\theta)\right)-x_{s}(\theta) \theta\right] f_{2}(\theta) d \theta .
\end{aligned}
$$

Note that

$$
\begin{aligned}
& \int_{\underline{\theta}}^{\bar{\theta}} \int_{\theta}^{\bar{\theta}}\left[x_{s}(t)\left[\frac{1-F(t)}{1-F(\theta)}\right]^{n-1} d t\right] f_{1}(\theta) d \theta \\
= & n \int_{\underline{\theta}}^{\bar{\theta}} \int_{\theta}^{\bar{\theta}}\left[x_{s}(t)\left[\frac{1-F(t)}{1-F(\theta)}\right]^{n-1} d t\right](1-F(\theta))^{n-1} f(\theta) d \theta \\
= & n \int_{\underline{\theta}}^{\bar{\theta}}\left[\int_{\theta}^{\bar{\theta}} x_{s}(t)(1-F(t))^{n-1} d t\right] f(\theta) d \theta---(1)
\end{aligned}
$$

Changing the order of integration we can write (1) as

$$
\begin{aligned}
& n \int_{\underline{\theta}}^{\bar{\theta}}\left[\int_{\theta}^{\bar{\theta}} x_{s}(t)(1-F(t))^{n-1} d t\right] f(\theta) d \theta \\
= & n \int_{\underline{\theta}}^{\bar{\theta}}\left[\int_{\underline{\theta}}^{t} f(\theta) d \theta\right] x_{s}(t)(1-F(t))^{n-1} d t \\
= & n \int_{\underline{\theta}}^{\bar{\theta}} F(t) x_{s}(t)(1-F(t))^{n-1} d t \\
= & n \int_{\underline{\theta}}^{\bar{\theta}} x_{s}(\theta) F(\theta)(1-F(\theta))^{n-1} d \theta----(2)
\end{aligned}
$$

Using (2) we now get

$$
\begin{aligned}
& \int_{\underline{\theta}}^{\bar{\theta}}\left(s\left(x_{s}(\theta)\right)-x_{s}(\theta) \theta-\int_{\theta}^{\bar{\theta}} x_{s}(t)\left[\frac{1-F(t)}{1-F(\theta)}\right]^{n-1}\right) f_{1}(\theta) d \theta \\
= & n \int_{\underline{\theta}}^{\bar{\theta}}\left[\left\{s\left(x_{s}(\theta)\right)-x_{s}(\theta) \theta\right\}(1-F(\theta))^{n-1} f(\theta)-x_{s}(\theta) F(\theta)(1-F(\theta))^{n-1}\right] d \theta \\
= & n \int_{\underline{\theta}}^{\bar{\theta}}\left[\left\{s\left(x_{s}(\theta)\right)-x_{s}(\theta) \theta\right\} f(\theta)-x_{s}(\theta) F(\theta)\right](1-F(\theta))^{n-1} d \theta----(3)
\end{aligned}
$$

Let

$$
K(\theta)=s\left(x_{s}(\theta)\right)-x_{s}(\theta) \theta .----(4)
$$


Then using the envelope theorem we get that

$$
K^{\prime}(\theta)=-x_{s}(\theta)---(4 a)
$$

From (3) we can now write

$$
\begin{aligned}
& n \int_{\underline{\theta}}^{\bar{\theta}}\left[\left\{s\left(x_{s}(\theta)\right)-x_{s}(\theta) \theta\right\} f(\theta)-x_{s}(\theta) F(\theta)\right](1-F(\theta))^{n-1} d \theta \\
= & n \int_{\underline{\theta}}^{\bar{\theta}}\left[K(\theta) f(\theta)+K^{\prime}(\theta) F(\theta)\right](1-F(\theta))^{n-1} d \theta=n \int_{\underline{\theta}}^{\bar{\theta}}(1-F(\theta))^{n-1} d(K(\theta) F(\theta)) \\
= & n\left[(1-F(\theta))^{n-1} K(\theta) F(\theta)\right]_{\underline{\theta}}^{\bar{\theta}}-n \int_{\underline{\theta}}^{\bar{\theta}} K(\theta) F(\theta) d(1-F(\theta))^{n-1} \\
= & n \int_{\underline{\theta}}^{\bar{\theta}} K(\theta) F(\theta)(n-1)(1-F(\theta))^{n-2} f(\theta) d \theta=\int_{\underline{\theta}}^{\bar{\theta}} K(\theta) f_{2}(\theta) d \theta \\
= & \int_{\underline{\theta}}^{\bar{\theta}}\left[s\left(x_{s}(\theta)\right)-x_{s}(\theta) \theta\right] f_{2}(\theta) d \theta \text { (using 4).--- - (5) }
\end{aligned}
$$

Hence, from (3) and (5) we get that

$$
\begin{aligned}
& \int_{\underline{\theta}}^{\bar{\theta}}\left(s\left(x_{s}(\theta)\right)-x_{s}(\theta) \theta-\int_{\theta}^{\bar{\theta}} x_{s}(t)\left[\frac{1-F(t)}{1-F(\theta)}\right]^{n-1}\right) f_{1}(\theta) d \theta \\
= & \int_{\underline{\theta}}^{\bar{\theta}}\left[s\left(x_{s}(\theta)\right)-x_{s}(\theta) \theta\right] f_{2}(\theta) d \theta .
\end{aligned}
$$

This completes proof of lemma 2

Proof of Proposition 1 We will first show that $\hat{\pi}_{1}^{C C}>\hat{\pi}_{1}^{D C}$. The rest of the inequalities can be proved using exactly similar logic.

Step 1 We will show that $x_{2}^{C C}(\theta)<x_{2}^{D C}(\theta)$ for all $\theta \in[\underline{\theta}, \bar{\theta}]$.

From lemma 1 we know that for any $\theta \in[\underline{\theta}, \bar{\theta}], x_{2}^{C C}(\theta)$ solves

$$
\begin{aligned}
x_{2}^{C C}(\theta) & =\arg \max _{x \in[0, \bar{x}]} s(x)-x \theta \\
& =\arg \max _{x \in[0, \bar{x}]}\left(q_{2}^{C C}\right)^{2}-x \theta \\
& =\arg \max _{x \in[0, \bar{x}]}\left(\frac{(a-c)(2-\gamma)+c\left(2 \lambda(x)-\gamma k \lambda\left(x_{1}^{C C}\right)\right)}{\left(4-\gamma^{2}\right)}\right)^{2}-x \theta----(6)
\end{aligned}
$$

We have (6) since, as noted earlier, in this particular case $s(x)$ is the gross second stage profit when both firms choose the new technology. Since $x_{s}(\theta) \in(0, \bar{x})$ for all $\theta \in[\underline{\theta}, \bar{\theta}], x_{2}^{C C}(\theta)$ is the solution in $x$ of the following (1OC and 2OC):

$$
\begin{aligned}
& \frac{\partial}{\partial x} {\left[\left(\frac{(a-c)(2-\gamma)+c\left(2 \lambda(x)-\gamma k \lambda\left(x_{1}^{C C}\right)\right)}{\left(4-\gamma^{2}\right)}\right)^{2}-x \theta\right]=0----(7 a) } \\
& \frac{\partial^{2}}{\partial x^{2}}\left[\left(\frac{(a-c)(2-\gamma)+c\left(2 \lambda(x)-\gamma k \lambda\left(x_{1}^{C C}\right)\right)}{\left(4-\gamma^{2}\right)}\right)^{2}-x \theta\right]<0----(7 b)
\end{aligned}
$$


(7a) and (7b) imply that $x_{2}^{C C}(\theta)$ is the solution in $x$ of the following:

$$
\begin{aligned}
\frac{4 c \lambda^{\prime}(x)}{\left(4-\gamma^{2}\right)^{2}}\left[(a-c)(2-\gamma)+2 c \lambda(x)-c \gamma k \lambda\left(x_{1}^{C C}\right)\right]-\theta & =0---(8 a) \\
\frac{4 c}{\left(4-\gamma^{2}\right)^{2}}\left[\lambda^{\prime \prime}(x)\left\{(a-c)(2-\gamma)+2 c \lambda(x)-c \gamma k \lambda\left(x_{1}^{C C}\right)\right\}+2 c\left(\lambda^{\prime}(x)\right)^{2}\right] & <0----(8 b)
\end{aligned}
$$

Since $k \geq 1$ and $\lambda^{\prime \prime}(x)<-\frac{2 c k\left(\lambda^{\prime}(x)\right)^{2}}{(a-c)(2-\gamma)}$ for all $x \in(0, \bar{x}),(8 \mathrm{~b})$ will always be satisfied.

Similarly, $x_{2}^{D C}(\theta)$ is the solution in $x$ of the following:

$$
\begin{aligned}
\frac{4 c \lambda^{\prime}(x)}{\left(4-\gamma^{2}\right)^{2}}[(a-c)(2-\gamma)+2 c \lambda(x)]-\theta & =0----(9 a) \\
\frac{4 c}{\left(4-\gamma^{2}\right)^{2}}\left[\lambda^{\prime \prime}(x)\{(a-c)(2-\gamma)+2 c \lambda(x)\}+2 c\left(\lambda^{\prime}(x)\right)^{2}\right] & <0----(9 b)
\end{aligned}
$$

Again, since $k \geq 1$ and $\lambda^{\prime \prime}(x)<-\frac{2 c k\left(\lambda^{\prime}(x)\right)^{2}}{(a-c)(2-\gamma)}$ for all $x \in(0, \bar{x}),(9 \mathrm{~b})$ will always be satisfied.

Note that since $x_{s}(\theta) \in(0, \bar{x})$ for all $\theta \in[\underline{\theta}, \bar{\theta}]$, we must have $x_{1}^{C C}(\theta)>0$. This means that LHS of (8a) is strictly lower than the LHS of (9a). Moreover, from (8b) and (9b) we get that the both the LHS of (8a) and (9a) are strictly decreasing in $x$. Hence, we get that $x_{2}^{C C}(\theta)<x_{2}^{D C}(\theta)$ for all $\theta \in[\underline{\theta}, \bar{\theta}]$.

Step 2 Using a logic similar to the one used in $(8 \mathrm{a})$ and $(8 \mathrm{~b})$ we get that $x_{1}^{C C}(\theta)$ is the solution in $x$ of the following:

$$
\begin{aligned}
\frac{4 c k \lambda^{\prime}(x)}{\left(4-\gamma^{2}\right)^{2}}\left[(a-c)(2-\gamma)+2 c k \lambda(x)-c \gamma \lambda\left(x_{2}^{C C}\right)\right]-\theta & =0---(10 a) \\
\frac{4 c k}{\left(4-\gamma^{2}\right)^{2}}\left[\lambda^{\prime \prime}(x)\left\{(a-c)(2-\gamma)+2 c k \lambda(x)-c \gamma \lambda\left(x_{2}^{C C}\right)\right\}+2 c k\left(\lambda^{\prime}(x)\right)^{2}\right] & <0----(10 b)
\end{aligned}
$$

As before, (10b) will always be satisfied. We know that $\hat{\pi}_{1}^{C C}=\int_{\underline{\theta}}^{\bar{\theta}}\left(\left[q_{1}^{C C}\left(x_{s}(\theta)\right)\right]^{2}-x_{s}(\theta) \theta\right) f_{2}(\theta) d \theta$. Note that when firm 1 computes its expected payoff $\left(\hat{\pi}_{1}^{C C}\right)$ it takes the expected value of $x_{2}^{C C}(\theta)$ as given. Let $\operatorname{Exp} . x_{2}^{C C}(\theta)=\hat{x}_{2}^{C C}$ and let $\operatorname{Exp} . x_{2}^{D C}(\theta)=\hat{x}_{2}^{D C}$. Then

$$
\begin{aligned}
& \hat{\pi}_{1}^{C C}= \int_{\underline{\theta}}^{\bar{\theta}}\left(\left[q_{1}^{C C}(\theta)\right]^{2}-x_{1}^{C C}(\theta) \theta\right) f_{2}(\theta) d \theta \\
&= \int_{\underline{\theta}}^{\bar{\theta}}\left[\left(\frac{(a-c)(2-\gamma)+c\left(2 \lambda\left(x_{1}^{C C}(\theta)\right)-\gamma \lambda\left(\hat{x}_{2}^{C C}\right)\right)}{\left(4-\gamma^{2}\right)}\right)^{2}-x_{1}^{C C}(\theta) \theta\right] f_{2}(\theta) d \theta----(11) \\
& \hat{\pi}_{1}^{D C}=\left(q_{1}^{D C}\right)^{2}=\left[\frac{(a-c)(2-\gamma)-c \lambda\left(\hat{x}_{2}^{D C}\right) \gamma}{\left(4-\gamma^{2}\right)}\right]^{2}---(12)
\end{aligned}
$$

From (11) and (12) we get

$$
\hat{\pi}_{1}^{C C}-\hat{\pi}_{1}^{D C}
$$




$$
\begin{gathered}
=\int_{\underline{\theta}}^{\bar{\theta}}\left[\left(\frac{(a-c)(2-\gamma)+c\left(2 \lambda\left(x_{1}^{C C}(\theta)\right)-\gamma \lambda\left(\hat{x}_{2}^{C C}\right)\right)}{\left(4-\gamma^{2}\right)}\right)^{2}-x_{1}^{C C}(\theta) \theta\right] f_{2}(\theta) d \theta \\
-\left[\frac{(a-c)(2-\gamma)-c \lambda\left(\hat{x}_{2}^{D C}\right) \gamma}{\left(4-\gamma^{2}\right)}\right]^{2} \\
=\int_{\underline{\theta}}^{\bar{\theta}}\left[\begin{array}{l}
\left.\left(\frac{(a-c)(2-\gamma)+c\left(2 \lambda\left(x_{1}^{C C}(\theta)\right)-\gamma \lambda\left(\hat{x}_{2}^{C C}\right)\right)}{\left(4-\gamma^{2}\right)}\right)^{2}\right] \\
-\left(\frac{(a-c)(2-\gamma)-c \lambda\left(\hat{x}_{2}^{D C}\right) \gamma}{\left(4-\gamma^{2}\right)}\right)^{2}-x_{1}^{C C}(\theta) \theta
\end{array}\right] f_{2}(\theta) d \theta----(13)
\end{gathered}
$$

Note that

$$
\begin{aligned}
& \left(\frac{(a-c)(2-\gamma)+c\left(2 k \lambda\left(x_{1}^{C C}(\theta)\right)-\gamma \lambda\left(\hat{x}_{2}^{C C}\right)\right)}{\left(4-\gamma^{2}\right)}\right)^{2}-\left(\frac{(a-c)(2-\gamma)-c \lambda\left(\hat{x}_{2}^{D C}\right) \gamma}{\left(4-\gamma^{2}\right)}\right)^{2}-x_{1}^{C C}(\theta) \theta \\
= & \frac{c}{\left(4-\gamma^{2}\right)^{2}}\left[\begin{array}{c}
2 k \lambda\left(x_{1}^{C C}(\theta)\right) \\
+\gamma\left(\lambda\left(\hat{x}_{2}^{D C}\right)-\lambda\left(\hat{x}_{2}^{C C}\right)\right)
\end{array}\right]\left[\begin{array}{c}
2(a-c)(2-\gamma) \\
+2 c k \lambda\left(x_{1}^{C C}(\theta)\right)-c \gamma\left(\lambda\left(\hat{x}_{2}^{D C}\right)+\lambda\left(\hat{x}_{2}^{C C}\right)\right)
\end{array}\right]-x_{1}^{C C}(\theta) \theta
\end{aligned}
$$

Using (10a) we get can write the above as

$$
\begin{aligned}
& \frac{c}{\left(4-\gamma^{2}\right)^{2}}\left[\begin{array}{c}
2 k \lambda\left(x_{1}^{C C}(\theta)\right) \\
+\gamma\left(\lambda\left(\hat{x}_{2}^{D C}\right)-\lambda\left(\hat{x}_{2}^{C C}\right)\right)
\end{array}\right]\left[\begin{array}{c}
2(a-c)(2-\gamma) \\
+2 c k \lambda\left(x_{1}^{C C}(\theta)\right)-c \gamma\left(\lambda\left(\hat{x}_{2}^{D C}\right)+\lambda\left(\hat{x}_{2}^{C C}\right)\right)
\end{array}\right] \\
& -\frac{4 c k x_{1}^{C C}(\theta) \lambda^{\prime}\left(x_{1}^{C C}(\theta)\right)}{\left(4-\gamma^{2}\right)^{2}}\left[(a-c)(2-\gamma)+2 c k \lambda\left(x_{1}^{C C}(\theta)\right)-c \gamma \lambda\left(\hat{x}_{2}^{C C}\right)\right]
\end{aligned}
$$

Hence, we get that

$$
\begin{aligned}
& \left(\frac{(a-c)(2-\gamma)+c\left(2 k \lambda\left(x_{1}^{C C}(\theta)\right)-\gamma \lambda\left(\hat{x}_{2}^{C C}\right)\right)}{\left(4-\gamma^{2}\right)}\right)^{2}-\left(\frac{(a-c)(2-\gamma)-c \lambda\left(\hat{x}_{2}^{D C}\right) \gamma}{\left(4-\gamma^{2}\right)}\right)^{2}-x_{1}^{C C}(\theta) \theta \\
= & \frac{c}{\left(4-\gamma^{2}\right)^{2}}\left[\begin{array}{c}
2 k \lambda\left(x_{1}^{C C}(\theta)\right) \\
+\gamma\left(\lambda\left(\hat{x}_{2}^{D C}\right)-\lambda\left(\hat{x}_{2}^{C C}\right)\right)
\end{array}\right]\left[\begin{array}{c}
2(a-c)(2-\gamma) \\
+2 c k \lambda\left(x_{1}^{C C}(\theta)\right)-c \gamma\left(\lambda\left(\hat{x}_{2}^{D C}\right)+\lambda\left(\hat{x}_{2}^{C C}\right)\right)
\end{array}\right] \\
& -\frac{4 c k x_{1}^{C C}(\theta) \lambda^{\prime}\left(x_{1}^{C C}(\theta)\right)}{\left(4-\gamma^{2}\right)^{2}}\left[(a-c)(2-\gamma)+2 c k \lambda\left(x_{1}^{C C}(\theta)\right)-c \gamma \lambda\left(x_{2}^{C C}\right)\right]---(14)
\end{aligned}
$$

Step 3 Now let

$$
\begin{aligned}
g(x)= & \frac{c}{\left(4-\gamma^{2}\right)^{2}}\left[\begin{array}{c}
2 k \lambda(x) \\
+\gamma\left(\lambda\left(\hat{x}_{2}^{D C}\right)-\lambda\left(\hat{x}_{2}^{C C}\right)\right)
\end{array}\right]\left[\begin{array}{c}
2(a-c)(2-\gamma) \\
+2 c k \lambda(x)-c \gamma\left(\lambda\left(\hat{x}_{2}^{D C}\right)+\lambda\left(\hat{x}_{2}^{C C}\right)\right)
\end{array}\right] \\
& -\frac{4 c k x \lambda^{\prime}(x)}{\left(4-\gamma^{2}\right)^{2}}\left[(a-c)(2-\gamma)+2 c k \lambda(x)-c \gamma \lambda\left(\hat{x}_{2}^{C C}\right)\right]----(15)
\end{aligned}
$$

From step 1 we know that $\hat{x}_{2}^{D C}>\hat{x}_{2}^{C C}$ and this implies that $\lambda\left(\hat{x}_{2}^{D C}\right)>\lambda\left(\hat{x}_{2}^{C C}\right)$. From the assumptions of our model we have $a>2 c, \gamma \in(0,1)$ and $k \lambda() \leq$.1 . Using these facts in (15) we get that

$$
g(0)>0----(16)
$$


Note that

$$
g^{\prime}(x)=4 x k\left[-\lambda^{\prime \prime}(x)\left\{(a-c)(2-\gamma)+2 c k \lambda(x)-c \gamma \lambda\left(\hat{x}_{2}^{C C}\right)\right\}-2 c k\left(\lambda^{\prime}(x)\right)^{2}\right]----
$$

Since $\lambda^{\prime \prime}(x)<-\frac{2 c k\left(\lambda^{\prime}(x)\right)^{2}}{(a-c)(2-\gamma)}$ for all $x \in(0, \bar{x})$ we get that

$$
g^{\prime}(x)>0 \text { for all } x \in(0, \bar{x})----(18
$$

From (17) and (18) we get that

$$
g(x)>0 \text { for all } x \in(0, \bar{x})----(19)
$$

This means for any $\theta \in[\underline{\theta}, \bar{\theta}]$

$$
\begin{gathered}
\qquad\left(x_{1}^{C C}(\theta)\right)>0 \Longrightarrow \\
\\
\quad \frac{c}{\left(4-\gamma^{2}\right)^{2}}\left[\begin{array}{c}
2 k \lambda\left(x_{1}^{C C}(\theta)\right) \\
+\gamma\left(\lambda\left(\hat{x}_{2}^{D C}\right)-\lambda\left(\hat{x}_{2}^{C C}\right)\right)
\end{array}\right]\left[\begin{array}{c}
2(a-c)(2-\gamma) \\
+2 c k \lambda\left(x_{1}^{C C}(\theta)\right)-c \gamma\left(\lambda\left(\hat{x}_{2}^{D C}\right)+\lambda\left(\hat{x}_{2}^{C C}\right)\right)
\end{array}\right] \\
\quad-\frac{4 c k x_{1}^{C C}(\theta) \lambda^{\prime}\left(x_{1}^{C C}(\theta)\right)}{\left(4-\gamma^{2}\right)^{2}}\left[(a-c)(2-\gamma)+2 c k \lambda\left(x_{1}^{C C}(\theta)\right)-c \gamma \lambda\left(x_{2}^{C C}\right)\right] \\
>0----(20)
\end{gathered}
$$

Using (20) in (14) we get that

$$
\begin{aligned}
& \left(\frac{(a-c)(2-\gamma)+c\left(2 \lambda\left(x_{1}^{C C}(\theta)\right)-\gamma \lambda\left(\hat{x}_{2}^{C C}\right)\right)}{\left(4-\gamma^{2}\right)}\right)^{2}-\left(\frac{(a-c)(2-\gamma)-c \lambda\left(\hat{x}_{2}^{D C}\right) \gamma}{\left(4-\gamma^{2}\right)}\right)^{2}-x_{1}^{C C}(\theta) \theta \\
> & 0----(21)
\end{aligned}
$$

Using (21) and (13) we get that $\hat{\pi}_{1}^{C C}-\hat{\pi}_{1}^{D C}>0$.

Proof of Lemma 3 We will prove it for the case where both firms choose the new technology. the other cases can be proved similarly. From lemma 1 we know that for any $\theta \in[\underline{\theta}, \bar{\theta}], x_{1}^{C C}(\theta)$ solves

$$
\begin{aligned}
x_{1}^{C C}(\theta) & =\arg \max _{x \in[0, \bar{x}]} s(x)-x \theta \\
& =\arg \max _{x \in[0, \bar{x}]}\left(q_{1}^{C C}\right)^{2}-x \theta \\
& =\arg \max _{x \in[0, \bar{x}]}\left(\frac{(a-c)(2-\gamma)+c\left(2 k \lambda(x)-\gamma \lambda\left(x_{2}^{C C}\right)\right)}{\left(4-\gamma^{2}\right)}\right)^{2}-x \theta----(22
\end{aligned}
$$

Now note that

$$
\begin{aligned}
\frac{\partial}{\partial x}\left[\left(q_{1}^{C C}\right)^{2}-x \theta\right] & =\frac{4 c k \lambda^{\prime}(x)}{\left(4-\gamma^{2}\right)^{2}}\left[(a-c)(2-\gamma)+2 c k \lambda(x)-c \gamma \lambda\left(x_{2}^{C C}\right)\right]-\theta---(23 a) \\
\frac{\partial^{2}}{\partial x^{2}}\left[\left(q_{1}^{C C}\right)^{2}-x \theta\right] & =\frac{4 c k}{\left(4-\gamma^{2}\right)^{2}}\left[\lambda^{\prime \prime}(x)\left\{\begin{array}{c}
(a-c)(2-\gamma) \\
+2 c k \lambda(x)-c \gamma \lambda\left(x_{2}^{C C}\right)
\end{array}\right\}+2 c k\left(\lambda^{\prime}(x)\right)^{2}\right]--(23 b)
\end{aligned}
$$


We have assumed that $x_{s}(\theta)>0$ for all $\theta \in[\underline{\theta}, \bar{\theta}]$. This means $\frac{\partial}{\partial x}\left[\left(q_{1}^{C C}\right)^{2}-x \theta\right]>0$ at $x=0$. Since $a>2 c, \gamma \in(0,1)$ and $k \lambda(x) \leq 1$ for all $x \in[0, \bar{x}]$ (by assumption), from (23b) we get that if $\lambda^{\prime \prime}(x) \geq 0$ then $\frac{\partial}{\partial x}\left[\left(q_{1}^{C C}\right)^{2}-x \theta\right]>0$ for all $x \in[0, \bar{x}]$. This means that $\frac{\partial}{\partial x}\left[\left(q_{1}^{C C}\right)^{2}-x \theta\right]>0$ for all $x \in(0, \bar{x})$. This implies $x_{1}^{C C}(\theta)=\bar{x}$ for all $\theta \in[\underline{\theta}, \bar{\theta}]$

Proof of Lemma 4 Let

$$
h(k)=(a-c)(2-\gamma)(k-1)+c \lambda(\bar{x})\left(k^{2}-k \gamma-1\right)----(24
$$

Then

$$
h^{\prime}(k)=(a-c)(2-\gamma)+c \lambda(\bar{x})(2 k-\gamma)----
$$

Since $k \geq 1$ and $\gamma \in(0,1)$ we have

$$
h(1)<0 \text { and } h^{\prime}(k)>0 \text { for all } k \geq 1----(26)
$$

(26) implies $\exists$ unique $\underline{k} \in(1, \infty)$ s.t. $h(\underline{k})=0$. This proves lemma 4 (i). Part (ii) of lemma 4 can be proved using similar logic.

Proof of Lemma 5 We know that $\hat{\pi}_{1}^{C C}=\int_{\underline{\theta}}^{\bar{\theta}}\left(\left[q_{1}^{C C}\left(x_{s}(\theta)\right)\right]^{2}-x_{s}(\theta) \theta\right) f_{2}(\theta) d \theta$ and $\hat{\pi}_{1}^{D C}=$ $\left(q_{1}^{D C}\right)^{2}$. From lemma 3 we get that when $\lambda^{\prime \prime}(x) \geq 0$ for all $x \in(0, \bar{x}), x_{1}^{C C}(\theta)=x_{2}^{C C}(\theta)=$ $x_{2}^{D C}(\theta)=\bar{x}$. Note that $z=\int_{\underline{\theta}}^{\bar{\theta}} f_{2}(\theta)$.

$$
\begin{aligned}
\hat{\pi}_{1}^{C C} & =\int_{\underline{\theta}}^{\bar{\theta}}\left(\left[q_{1}^{C C}(\theta)\right]^{2}-x_{1}^{C C}(\theta) \theta\right) f_{2}(\theta) d \theta \\
& =\int_{\underline{\theta}}^{\bar{\theta}}\left[\left(\frac{(a-c)(2-\gamma)+c(2 k \lambda(\bar{x})-\gamma \lambda(\bar{x}))}{\left(4-\gamma^{2}\right)}\right)^{2}-\bar{x} \theta\right] f_{2}(\theta) d \theta \\
& =\left(\frac{(a-c)(2-\gamma)+c(2 k \lambda(\bar{x})-\gamma \lambda(\bar{x}))}{\left(4-\gamma^{2}\right)}\right)^{2}-z \bar{x}---(27) \\
& \hat{\pi}_{1}^{D C}=\left(q_{1}^{D C}\right)^{2}=\left[\frac{(a-c)(2-\gamma)-c \lambda(\bar{x}) \gamma}{\left(4-\gamma^{2}\right)}\right]^{2}---(28)
\end{aligned}
$$

Using (27) and (28) we get that

$$
\begin{aligned}
\hat{\pi}_{1}^{C C}-\hat{\pi}_{1}^{D C}= & \left(\frac{(a-c)(2-\gamma)+c(2 \lambda(\bar{x})-\gamma \lambda(\bar{x}))}{\left(4-\gamma^{2}\right)}\right)^{2}-z \bar{x} \\
& -\left[\frac{(a-c)(2-\gamma)-c \lambda(\bar{x}) \gamma}{\left(4-\gamma^{2}\right)}\right]^{2} \\
= & \frac{4 c k \lambda(\bar{x})}{\left(4-\gamma^{2}\right)^{2}}[(a-c)(2-\gamma)+c \lambda(\bar{x})(k-\gamma)]-z \bar{x}----
\end{aligned}
$$


Using similar methods we can compute $\hat{\pi}_{2}^{C C}-\hat{\pi}_{2}^{C D}, \hat{\pi}_{1}^{C D}-\hat{\pi}_{1}^{D D}$ and $\hat{\pi}_{2}^{D C}-\hat{\pi}_{2}^{D D}$. We list all of them below.

$$
\begin{aligned}
\hat{\pi}_{1}^{C C}-\hat{\pi}_{1}^{D C} & =\frac{4 c k \lambda(\bar{x})}{\left(4-\gamma^{2}\right)^{2}}[(a-c)(2-\gamma)+c \lambda(\bar{x})(k-\gamma)]-z \bar{x}---(30 a) \\
\hat{\pi}_{2}^{C C}-\hat{\pi}_{2}^{C D} & =\frac{4 c \lambda(\bar{x})}{\left(4-\gamma^{2}\right)^{2}}[(a-c)(2-\gamma)+c \lambda(\bar{x})(1-k \gamma)]-z \bar{x}----(30 b) \\
\hat{\pi}_{1}^{C D}-\hat{\pi}_{1}^{D D} & =\frac{4 c k \lambda(\bar{x})}{\left(4-\gamma^{2}\right)^{2}}[(a-c)(2-\gamma)+c k \lambda(\bar{x})]-z \bar{x}---(30 c) \\
\hat{\pi}_{2}^{D C}-\hat{\pi}_{2}^{D D} & =\frac{4 c \lambda(\bar{x})}{\left(4-\gamma^{2}\right)^{2}}[(a-c)(2-\gamma)+c \lambda(\bar{x})]-z \bar{x}---(30 d)
\end{aligned}
$$

Note that $\gamma \in(0,1)$. Then, for all $k>1$ by inspecting (30a) to (30d) we get that $\hat{\pi}_{1}^{C D}-\hat{\pi}_{1}^{D D}$ is strictly greater than $\hat{\pi}_{1}^{C C}-\hat{\pi}_{1}^{D C}, \hat{\pi}_{2}^{C C}-\hat{\pi}_{2}^{C D}$ and $\hat{\pi}_{2}^{D C}-\hat{\pi}_{2}^{D D}$. Similarly $\hat{\pi}_{2}^{C C}-\hat{\pi}_{2}^{C D}$ is strictly lower than $\hat{\pi}_{1}^{C C}-\hat{\pi}_{1}^{D C}, \hat{\pi}_{1}^{C D}-\hat{\pi}_{1}^{D D}$ and $\hat{\pi}_{2}^{D C}-\hat{\pi}_{2}^{D D}$. Now we will compare $\hat{\pi}_{1}^{C C}-\hat{\pi}_{1}^{D C}$ and $\hat{\pi}_{2}^{D C}-\hat{\pi}_{2}^{D D}$. Note that

$$
\begin{aligned}
& \left(\hat{\pi}_{1}^{C C}-\hat{\pi}_{1}^{D C}\right)-\left(\hat{\pi}_{2}^{D C}-\hat{\pi}_{2}^{D D}\right) \\
= & \frac{4 c \lambda(\bar{x})}{\left(4-\gamma^{2}\right)^{2}}\left[(a-c)(2-\gamma)(k-1)+c \lambda(\bar{x})\left(k^{2}-k \gamma-1\right)\right]----(31
\end{aligned}
$$

Using (24) we can write

$$
\left(\hat{\pi}_{1}^{C C}-\hat{\pi}_{1}^{D C}\right)-\left(\hat{\pi}_{2}^{D C}-\hat{\pi}_{2}^{D D}\right)=\frac{4 c \lambda(\bar{x})}{\left(4-\gamma^{2}\right)^{2}} h(k)----
$$

From (26) and from the proof of lemma 4 we know that $h(1)<0, h(\underline{k})=0$ and $h^{\prime}(k)>0$ for all $k \geq 1$. This means for all $k \in(1, \underline{k})$ we have $\left(\hat{\pi}_{1}^{C C}-\hat{\pi}_{1}^{D C}\right)<\left(\hat{\pi}_{2}^{D C}-\hat{\pi}_{2}^{D D}\right)$ and for all $k \in(\underline{k}, \infty)$ we have $\left(\hat{\pi}_{1}^{C C}-\hat{\pi}_{1}^{D C}\right)>\left(\hat{\pi}_{2}^{D C}-\hat{\pi}_{2}^{D D}\right)$. Combining this with our previous discussion we get that if $k \in(1, \underline{k})$ then

$$
\hat{\pi}_{1}^{C D}-\hat{\pi}_{1}^{D D}>\hat{\pi}_{2}^{D C}-\hat{\pi}_{2}^{D D}>\hat{\pi}_{1}^{C C}-\hat{\pi}_{1}^{D C}>\hat{\pi}_{2}^{C C}-\hat{\pi}_{2}^{C D}
$$

And, if $k \in(\underline{k}, \infty)$ then

$$
\hat{\pi}_{1}^{C D}-\hat{\pi}_{1}^{D D}>\hat{\pi}_{1}^{C C}-\hat{\pi}_{1}^{D C}>\hat{\pi}_{2}^{D C}-\hat{\pi}_{2}^{D D}>\hat{\pi}_{2}^{C C}-\hat{\pi}_{2}^{C D}
$$

This proves parts (i) and (ii) of lemma 5 .

Routine computations show that

$$
\begin{aligned}
\hat{B}_{1}^{C C}-\hat{B}_{1}^{D C} & =\frac{c k \lambda(\bar{x})\left(2-\gamma^{2}\right)}{\left(1-\gamma^{2}\right)\left(4-\gamma^{2}\right)^{2}}\left[\begin{array}{c}
2(a-c)\left(2-\gamma-\gamma^{2}\right) \\
+c k \lambda(\bar{x})\left(2-\gamma^{2}\right)-2 c \gamma \lambda(\bar{x})
\end{array}\right]-z \bar{x}----(33 a) \\
\hat{B}_{2}^{C C}-\hat{B}_{2}^{C D} & =\frac{c \lambda(\bar{x})\left(2-\gamma^{2}\right)}{\left(1-\gamma^{2}\right)\left(4-\gamma^{2}\right)^{2}}\left[\begin{array}{c}
2(a-c)\left(2-\gamma-\gamma^{2}\right) \\
+c \lambda(\bar{x})\left(2-\gamma^{2}\right)-2 c k \gamma \lambda(\bar{x})
\end{array}\right]-z \bar{x}----(33 b) \\
\hat{B}_{1}^{C D}-\hat{B}_{1}^{D D} & =\frac{c k \lambda(\bar{x})\left(2-\gamma^{2}\right)}{\left(1-\gamma^{2}\right)\left(4-\gamma^{2}\right)^{2}}\left[2(a-c)\left(2-\gamma-\gamma^{2}\right)+c k \lambda(\bar{x})\left(2-\gamma^{2}\right)\right]-z \bar{x}--(33 c) \\
\hat{B}_{2}^{D C}-\hat{B}_{2}^{D D} & =\frac{c \lambda(\bar{x})\left(2-\gamma^{2}\right)}{\left(1-\gamma^{2}\right)\left(4-\gamma^{2}\right)^{2}}\left[2(a-c)\left(2-\gamma-\gamma^{2}\right)+c \lambda(\bar{x})\left(2-\gamma^{2}\right)\right]-z \bar{x}--(33 d)
\end{aligned}
$$


Note that $\gamma \in(0,1)$. Then, for all $k>1$ by inspecting (30a) to (30d) we get that $\hat{B}_{1}^{C D}-\hat{B}_{1}^{D D}$ is strictly greater than $\hat{B}_{1}^{C C}-\hat{B}_{1}^{D C}, \hat{B}_{2}^{C C}-\hat{B}_{2}^{C D}$ and $\hat{B}_{2}^{D C}-\hat{B}_{2}^{D D}$. Similarly $\hat{B}_{2}^{C C}-\hat{B}_{2}^{C D}$ is strictly lower than $\hat{B}_{1}^{C C}-\hat{B}_{1}^{D C}, \hat{B}_{1}^{C D}-\hat{B}_{1}^{D D}$ and $\hat{B}_{2}^{D C}-\hat{B}_{2}^{D D}$. Using a logic exactly similar to one used before we can show that for all $k \in(1, \underline{\kappa})$ we have $\left(\hat{B}_{1}^{C C}-\hat{B}_{1}^{D C}\right)<\left(\hat{B}_{2}^{D C}-\hat{B}_{2}^{D D}\right)$ and for all $k \in(\underline{\kappa}, \infty)$ we have $\left(\hat{B}_{1}^{C C}-\hat{B}_{1}^{D C}\right)>\left(\hat{B}_{2}^{D C}-\hat{B}_{2}^{D D}\right)$. Combining this with our previous discussion we get that if $k \in(1, \underline{\kappa})$ then

$$
\hat{B}_{1}^{C D}-\hat{B}_{1}^{D D}>\hat{B}_{2}^{D C}-\hat{B}_{2}^{D D}>\hat{B}_{1}^{C C}-\hat{B}_{1}^{D C}>\hat{B}_{2}^{C C}-\hat{B}_{2}^{C D} .
$$

And, if $k \in(\underline{\kappa}, \infty)$ then

$$
\hat{B}_{1}^{C D}-\hat{B}_{1}^{D D}>\hat{B}_{1}^{C C}-\hat{B}_{1}^{D C}>\hat{B}_{2}^{D C}-\hat{B}_{2}^{D D}>\hat{B}_{2}^{C C}-\hat{B}_{2}^{C D} .
$$

This proves parts (iii) and (iv) of lemma 5 .

Proof of Proposition 3 We know that (see the discussion before the statement of proposition 3)

$$
\begin{gathered}
\hat{\pi}_{2}^{C C}-\hat{\pi}_{2}^{C D} \geq 0 \Longleftrightarrow \frac{4 c \lambda(\bar{x})}{\left(4-\gamma^{2}\right)^{2}}[(a-c)(2-\gamma)+c \lambda(\bar{x})(1-k \gamma)] \geq \bar{x} z \\
\hat{B}_{2}^{C C}-\hat{B}_{2}^{C D} \geq 0 \Longleftrightarrow \frac{c \lambda(\bar{x})\left(2-\gamma^{2}\right)}{\left(1-\gamma^{2}\right)\left(4-\gamma^{2}\right)^{2}}\left[2(a-c)\left(2-\gamma-\gamma^{2}\right)+c \lambda(\bar{x})\left(2-\gamma^{2}-2 k \gamma\right)\right] \geq \bar{x} z
\end{gathered}
$$

Using the above fact and using lemma 5 we get for all $k \in(1, \infty)$

$$
\begin{gathered}
\hat{\pi}_{1}^{C D}-\hat{\pi}_{1}^{D D}, \hat{\pi}_{2}^{D C}-\hat{\pi}_{2}^{D D}, \hat{\pi}_{1}^{C C}-\hat{\pi}_{1}^{D C}, \hat{\pi}_{2}^{C C}-\hat{\pi}_{2}^{C D}>0---(34 a) \\
\hat{B}_{1}^{C D}-\hat{B}_{1}^{D D}, \hat{B}_{2}^{D C}-\hat{B}_{2}^{D D}, \hat{B}_{1}^{C C}-\hat{B}_{1}^{D C}, \hat{B}_{2}^{C C}-\hat{B}_{2}^{C D}>0---(34 b)
\end{gathered}
$$

Therefore, from (34a) (or 34b) and the payoff matrix in section 5 we get that both firms adopting the new technology is the unique subgame perfect equilibrium when firms engage in quantity (or price) competition in the second stage. That is, the equilibrium outcome in the first stage is $(C, C)$.

Proof of Proposition 4 We know that (see the discussion before the statement of proposition $3)$

$$
\begin{aligned}
\hat{\pi}_{1}^{C D}-\hat{\pi}_{1}^{D D} & \leq 0 \Longleftrightarrow \frac{4 c k \lambda(\bar{x})}{\left(4-\gamma^{2}\right)^{2}}[(a-c)(2-\gamma)+c k \lambda(\bar{x})] \leq \bar{x} z \\
\hat{B}_{1}^{C D}-\hat{B}_{1}^{D D} & \leq 0 \Longleftrightarrow \frac{c k \lambda(\bar{x})\left(2-\gamma^{2}\right)}{\left(1-\gamma^{2}\right)\left(4-\gamma^{2}\right)^{2}}\left[2(a-c)\left(2-\gamma-\gamma^{2}\right)+c k \lambda(\bar{x})\left(2-\gamma^{2}\right)\right] \leq \bar{x} z
\end{aligned}
$$

Using the above fact and using lemma 5 we get for all $k \in(1, \infty)$

$$
\begin{gathered}
\hat{\pi}_{1}^{C D}-\hat{\pi}_{1}^{D D}, \hat{\pi}_{2}^{D C}-\hat{\pi}_{2}^{D D}, \hat{\pi}_{1}^{C C}-\hat{\pi}_{1}^{D C}, \hat{\pi}_{2}^{C C}-\hat{\pi}_{2}^{C D}<0----(35 a) \\
\hat{B}_{1}^{C D}-\hat{B}_{1}^{D D}, \hat{B}_{2}^{D C}-\hat{B}_{2}^{D D}, \hat{B}_{1}^{C C}-\hat{B}_{1}^{D C}, \hat{B}_{2}^{C C}-\hat{B}_{2}^{C D}
\end{gathered}
$$

Therefore, from (35a) (or 35b) and the payoff matrix in section 5 we get that both firms not adopting the new technology is the unique subgame perfect equilibrium when firms engage in quantity (or price) competition in the second stage. That is, the equilibrium outcome in the first stage is $(D, D)$. 
Proof of lemma 6 Note that

$$
\begin{aligned}
& \left(\hat{\pi}_{1}^{C D}-\hat{\pi}_{1}^{D D}\right)-\left(\hat{B}_{1}^{C D}-\hat{B}_{1}^{D D}\right) \\
= & \frac{c k \lambda(\bar{x}) \gamma^{3}}{\left(1-\gamma^{2}\right)\left(4-\gamma^{2}\right)^{2}}[2(a-c)(1-\gamma)-c k \gamma \lambda(\bar{x})]----(36
\end{aligned}
$$

Let

$$
l(\gamma)=2(a-c)(1-\gamma)-c k \gamma \lambda(\bar{x})----(37)
$$

Note that

$$
l(0)=2(a-c)>0, l(1)=-c k \lambda(\bar{x})<0 \text { and } l^{\prime}(\gamma)<0 \text { for all } \gamma \in(0,1)----
$$

From (37) and (38) we clearly get that

$$
\begin{aligned}
& l(\gamma)>0 \text { if } \gamma \in\left(0, \frac{2 a-2 c}{2 a-2 c+c k \lambda(\bar{x})}\right) \text { and } \\
& l(\gamma)<0 \text { if } \gamma \in\left(\frac{2 a-2 c}{2 a-2 c+c k \lambda(\bar{x})}, 1\right)----
\end{aligned}
$$

From (36) we know that

$$
\left(\hat{\pi}_{1}^{C D}-\hat{\pi}_{1}^{D D}\right)-\left(\hat{B}_{1}^{C D}-\hat{B}_{1}^{D D}\right)=\frac{c k \lambda(\bar{x}) \gamma^{3}}{\left(1-\gamma^{2}\right)\left(4-\gamma^{2}\right)^{2}} l(\gamma)----(40)
$$

using (39) and (40) we get that

$$
\begin{aligned}
& \left(\hat{\pi}_{1}^{C D}-\hat{\pi}_{1}^{D D}\right)-\left(\hat{B}_{1}^{C D}-\hat{B}_{1}^{D D}\right)>0 \text { if } \gamma \in\left(0, \frac{2 a-2 c}{2 a-2 c+c k \lambda(\bar{x})}\right) \text { and } \\
& \left(\hat{\pi}_{1}^{C D}-\hat{\pi}_{1}^{D D}\right)-\left(\hat{B}_{1}^{C D}-\hat{B}_{1}^{D D}\right)<0 \text { if } \gamma \in\left(\frac{2 a-2 c}{2 a-2 c+c k \lambda(\bar{x})}, 1\right)----(41)
\end{aligned}
$$

It maybe recalled that $\gamma \in(0, \underline{\gamma})$ where $\underline{\gamma}=\frac{-a+\sqrt{9 a^{2}+8 c^{2}-16 a c}}{2(a-c)}$. We cannot compare $\frac{2 a-2 c}{2 a-2 c+c k \lambda(\bar{x})}$ and $\frac{-a+\sqrt{9 a^{2}+8 c^{2}-16 a c}}{2(a-c)}$. So, using (41) we can state that if $\frac{2 a-2 c}{2 a-2 c+c k \lambda(\bar{x})}<\underline{\gamma}$, then for $\gamma \in\left(0, \frac{2 a-2 c}{2 a-2 c+c k \lambda(\bar{x})}\right)$ we get $\hat{\pi}_{1}^{C D}-\hat{\pi}_{1}^{D D}>\hat{B}_{1}^{C D}-\hat{B}_{1}^{D D}$ and for $\gamma \in\left(\frac{2 a-2 c}{2 a-2 c+c k \lambda(\bar{x})}, \underline{\gamma}\right)$ we get $\hat{\pi}_{1}^{C D}-\hat{\pi}_{1}^{D D}<\hat{B}_{1}^{C D}-\hat{B}_{1}^{D D}$.

Proof of Lemma 7 Note that

$$
\begin{aligned}
& \left(\hat{\pi}_{2}^{C C}-\hat{\pi}_{2}^{C D}\right)-\left(\hat{B}_{2}^{C C}-\hat{B}_{2}^{C D}\right) \\
= & \frac{c \gamma^{3} \lambda(\bar{x})}{\left(1-\gamma^{2}\right)\left(4-\gamma^{2}\right)^{2}}[2(a-c)(1-\gamma)+c \lambda(\bar{x})(2 k-\gamma)]----
\end{aligned}
$$

Since $a>2 c, \gamma \in(0, \underline{\gamma})$ where $\underline{\gamma}<1$ and $k \geq 1$ the RHS of (42) is strictly positive. Hence, $\left(\hat{\pi}_{2}^{C C}-\hat{\pi}_{2}^{C D}\right)>\left(\hat{B}_{2}^{C C}-\hat{B}_{2}^{C D}\right)$ for all $k \geq 1$ and for all $\gamma \in(0, \underline{\gamma})$ 